\title{
Random Sampling for Continuous Streams with Arbitrary Updates
}

\author{
Yufei Tao ${ }^{\S} \quad$ Xiang Lian ${ }^{\dagger} \quad$ Dimitris Papadias $^{\dagger} \quad$ Marios Hadjieleftheriou $^{\ddagger}$ \\ ${ }^{\S}$ Department of Computer Science and Engineering ${ }^{\dagger}$ Department of Computer Science and Engineering \\ Chinese University of Hong Kong \\ Sha Tin, New Territories, Hong Kong \\ taoyf@cse.cuhk.edu.hk \\ Hong Kong University of Science and Technology \\ AT\&T Labs \\ Clear Water Bay, Hong Kong \\ \{xlian, dimitris\}@cse.ust.hk \\ Florham Park, NJ, USA \\ marioh@research.att.com
}

\begin{abstract}
The existing random sampling methods have at least one of the following disadvantages: they (i) are applicable only to certain update patterns, (ii) entail large space overhead, or (iii) incur prohibitive maintenance cost. These drawbacks prevent their effective application in stream environments (where a relation is updated by a large volume of insertions and deletions that may arrive in any order), despite the considerable success of random sampling in conventional databases.

Motivated by this, we develop several fully-dynamic algorithms for obtaining random samples from individual relations, and from the join result of two tables. Our solutions can handle any update pattern with small space and computational overhead. We also present an in-depth analysis that provides valuable insight into the characteristics of alternative sampling strategies and leads to precision guarantees. Extensive experiments validate our theoretical findings and demonstrate the efficiency of our techniques in practice.
\end{abstract}

IEEE Transactions on Knowledge and Data Engineering (TKDE), 19(1), 96-110, 2007. Keywords: Sampling, Selectivity Estimation

\section{Contact Author:}

Yufei Tao

Department of Computer Science and Engineering

Chinese University of Hong Kong

Sha Tin, New Territories, Hong Kong

Tel: $+852-26098437$; Fax: $+852-26035024$

Http://www.cse.cuhk.edu.hk/ taoyf 


\section{INTRODUCTION}

A general stream relation receives a large number of updates per time unit, which include tuple insertions and deletions that may arrive in any order. Specifically, each I-command has the form $\{\mathrm{I}, i d$, $\left.S_{A}\right\}$, where the first field is a tag indicating "insertion", the second denotes the id of the tuple being inserted, and $S_{A}$ corresponds to the set of the remaining attributes of the tuple. Similarly, a D-command $\{\mathrm{D}, i d\}$ removes the tuple with a specified id. The content of a relation includes all the tuples that were inserted, but have not been removed; the cardinality of the relation is the number of such tuples.

We consider that all tuples currently in a relation have distinct $i d$ 's. Specifically, the $i d$ in each Icommand should not be identical to the $i d$ of any existing tuple in the relation. However, provided that the previous tuple with an $i d$ has been deleted, a tuple with the same $i d$ can be inserted. In other words, multiple tuples with the same $i d$ may be inserted throughout the history, but at any moment, there can be only one tuple with this $i d$.

We address two fundamental problems of approximate processing. Given a single relation $T$, the first one aims at providing accurate answers to queries of the form:

\section{Q1: SELECT COUNT(*) FROM $T$ WHERE $\theta_{\text {any }}$}

Q1 is a counting query with arbitrary conditions $\theta_{\text {any }}$ in the WHERE clause. We assume no a priori knowledge about $\theta_{\text {any }}$, which excludes potential solutions $[16,17]$ that rely on special counting "sketches" (including histograms, wavelets, etc.) for certain attributes (or their combinations). Specifically, although these methods can produce accurate results on the pre-processed columns, they are not useful for general ad-hoc queries involving other predicates. Random-sampling techniques, on the other hand, constitute a natural methodology for this problem because, by keeping all attributes of the sampled tuples, it is possible to support any predicate $\theta_{\text {any }}$ with good accuracy guarantees.

The second problem tackled in this paper is to accurately predict the join size of two stream relations $T_{a}$ and $T_{b}$ : 


\section{Q2: SELECT COUNT(*) FROM $T_{a}, T_{b}$ WHERE $\theta_{\text {all }}$ and $\theta_{\text {any }}$}

$\theta_{\text {all }}$ includes a set of registered conditions common in all Q2 queries, which differ in their own formulation of $\theta_{a n y}$ (an arbitrary predicate). As an example, assume that $T_{a}$ has attributes $\left(i d, A_{a}\right), T_{b}$ has $\left(i d, A_{b}\right)$, and $\theta_{\text {all }}$ is $T_{a} . i d=T_{b} . i d$. Then, every possible Q2 query contains this equi-join condition, but can also include a predicate $\theta_{\text {any }}$ on individual columns of a single table (e.g., $\left.T_{a} . A_{a}>10\right)$, or both tables (e.g., $\left.T_{a} \cdot A_{a}+T_{b} \cdot A_{b}>100\right)$. Equivalently, the set of records counted in a Q2 query is a subset of the results of joining $T_{a}$ and $T_{b}$ using only the condition $T_{a} . i d=T_{b} . i d$. Our goal is to process Q1 and Q2 accurately using at most $M$ random samples, where $M$ is (by far) lower than the size of the database.

Query types Q1 and Q2 are important to a large number of applications. For example, consider a relation with schema $P R($ stock-id, price), where each tuple records the current price of a stock. Whenever a stock's price changes, a D-command streams in, removing the obsolete tuple of the stock; then, an Icommand immediately follows, inserting a new tuple carrying the updated price. Obviously, unlike the sliding-window stream model [5], the order that the tuples in $P R$ are inserted is most likely not equivalent to the order that they are deleted. A Q1 query in this context may retrieve the number of stocks whose prices qualify a certain predicate.

To motivate Q2 queries, assume that we have another stream relation TO(stock-id, turnover), which captures the current turnovers of the stocks. As with $P R, T O$ is updated with continuously arriving I- and D-commands, generated by the buying/selling of any stock. Note that the streams corresponding to $P R$ and $T O$ are typically separate, because they are usually generated by different agents. In this case, to study the statistic relationship between prices and turnovers, a user needs to join $P R$ and $T O$ together with an equality-condition on stock-id, and then apply other predicates on price and turnover. Such operations form a Q2 query, where $\theta_{\text {all }}$ is the equality-condition and $\theta_{\text {any }}$ corresponds to the other predicates.

We are interested in solutions that incur small computational overhead for processing each incoming command, as opposed to methods (e.g., the counting sample reviewed in the next section) that have low 
amortized cost but poor worst-case performance for individual updates. For data streams with high record arrival rate, spending considerable time on any tuple necessarily delays a large number of subsequent records, which need to be stored in a system buffer. When the size of this buffer is exceeded, some tuples must be discarded (i.e., load shedding [25]), in which case obtaining a truly-random sample set is impossible. For instance, in the stock-trading application mentioned earlier, shedding an update command causes the price of a stock to be inaccurate. Furthermore, shedding a D-command may even lead to duplicate tuples for the same stock.

As elaborated in Section 2, the existing sampling algorithms have at least one of the following disadvantages: (i) they rely on certain assumptions on data updates (e.g., only insertions are allowed, or tuples must be deleted according to the insertion order), (ii) they require considerable space (e.g., the underlying relations must be fully materialized for re-sampling), or (iii) they incur prohibitive maintenance overhead (e.g., they must periodically scan the entire sample set). These problems prevent their effective deployment on data stream applications, despite the success of random sampling in conventional databases.

In this paper, we develop sampling algorithms that are fully dynamic (supporting any sequence of insertions and deletions), efficient (processing each update with very low space and computational overhead), and accurate (producing approximate answers for Q1 and Q2 queries with small error). Specifically, for single relations, our methods significantly improve the well-known reservoir sampling and counting sample approaches in the presence of intensive updates. For join results, we propose the first sampling algorithm that enables effective approximate processing on streams containing arbitrary update requests (the previous solutions [24] discuss only the special case of sliding windows). In addition, we present an in-depth analysis that provides valuable insight into the characteristics of alternative solutions. Extensive experiments validate our theoretical findings and confirm the efficiency of the proposed techniques in practice.

The rest of the paper is organized as follows. Section 2 reviews previous work that is directly related 
to ours. Sections 3 and 4 present algorithms for sampling single relations, and analyze their effectiveness on Q1 queries. Section 5 focuses on sampling join results for approximate Q2 processing. Section 6 contains the experimental results, and Section 7 concludes the paper with directions for future work.

\section{RELATED WORK}

Section 2.1 first reviews approaches for sampling a single relation in the presence of updates, and clarifies their problems. Then, Section 2.2 discusses algorithms for sampling the join result of multiple tables.

\subsection{Sampling a Single Relation}

Sampling from a static table with $n$ tuples is straightforward. Specifically, a sequence number can be assigned to each record (i.e., the first tuple has number 1, the second 2, and so on). To obtain $s$ samples, we only need to randomly generate $s$ distinct values in the range $[1, n]$, and select the tuples with these sequence numbers. It is more difficult, however, to maintain the randomness of the sample set when new records are inserted into the table, or existing ones are removed. A naïve solution would re-sample the relation as described above whenever its content changes. Obviously, this method is impractical since a re-sampling process may require accessing a large number of disk pages. Reservoir sampling and counting sample aim at solving these problems.

Reservoir Sampling. The first reservoir algorithms [26, 22] in the database context maintain an array $R S$ with maximum size $s$ (the target sample size), which is initially empty. The first $s$ tuples are directly added into $R S$, after which the array becomes full. For each subsequent insertion, a random integer $x$ is generated in the range $[1, n]$, where $n$ is the number of insertions handled so far ( $n$ continuously grows with time). If $x$ is larger than $s$, the incoming tuple is ignored; otherwise $(x \leq s)$, it is recorded at the $x$-th position of $R S$, replacing the sample that was originally there. It can be shown [26] that, at any time, the tuples in $R S$ constitute a random sample set of the current content of the dataset $T$. Jermaine et al. [21] 
present an alternative reservoir technique to manage sample sets whose sizes exceed the capacity of the available memory. Similar to $[26,12]$, this approach supports only insertions.

Gibbons et al. [14] propose an extension, referred to as delete-at-will in the sequel, for producing a random sample set in the presence of deletions. Let $s$ be the current size of the sample set $R S$ and $n$ the cardinality of $T$. If the tuple $t$ being deleted does not belong to $R S$, the sample set remains unchanged. If $t$ is found in $R S$, it is removed, after which the size of $R S$ becomes $s-1$. In either case (whether $t$ appears in $R S$ or not), the cardinality of the relation $T$ changes to $n-1$ to reflect the removal of $t$ from $T$. The handling of insertions is similar to the reservoir technique. Assume, for instance, that after a sample is deleted (i.e., the sample size is $s-1$ and the data cardinality is $n-1$ ) a new record arrives. A random integer $x$ is generated in the range $[1, n-1]$ and if $x$ is larger than $s-1$, the incoming tuple is ignored; otherwise $(x \leq s-1)$, it is recorded at the $x$-th position of $R S$, replacing the sample that was originally there. Gibbons et al. [14] prove that the resulting $R S$ is still random with respect to the remaining tuples in $T$.

The problem with delete-at-will is that the sample set gradually shrinks with time, and eventually ceases to be useful (e.g., it can no longer provide accurate selectivity estimation). Therefore, $T$ must be scanned so that a sufficient number of samples are retrieved again. This requires retaining all the tuples that have been inserted but not yet deleted, which is impossible in stream environments. In Section 3 we propose $\mathrm{R}^{*}$, an improved reservoir algorithm that supports deletions without decreasing the sample size.

Counting Sample. Counting sample (CS) [13] can produce a random sample set for any sequence of insertions and deletions without re-sampling the base relation, even in the existence of duplicate tuples (consequently, it is trivially applicable to conventional tables where each record is different). Specifically, CS maintains a list $R S$ (with maximum size $s$ ) of elements in the form ${ }^{1}\{t, c\}$, where $c$ is a counter that summarizes the number of identical records $t$ in the sample set. Furthermore, a variable $\tau$,

\footnotetext{
${ }^{1}$ CS adopts a slightly more complex element representation to minimize the space consumption. Since the basic idea is the same, we ignore this difference here for simplicity.
} 
initially set to 1 , is used to control the probability that a record is sampled.

To handle an incoming tuple $t$, CS first probes the existing sample set to see if $t$ has been included before. If yes, the counter $c$ in the corresponding pair $\{t, c\}$ is increased by one, and the insertion terminates. Otherwise ( $t$ is not in $R S)$, CS tosses a coin $c_{1}$ with probability $1 / \tau$ head, and discards $t$ if $c_{1}$ tails. Alternatively ( $c_{1}$ heads), the algorithm includes into $R S$ a new entry $\{t, 1\}$. If $R S$ does not overflow (i.e., it contains no more than $s$ elements), the insertion is completed. In case of an overflow, a rejecting pass is performed to expunge some existing samples from $R S$.

Specifically, at the beginning of the rejecting pass, a number $\tau^{\prime}$ larger than the current $\tau$ (that governs the sampling probability, as mentioned earlier) is chosen. Then, for each element $\{t, c\}$ in $R S$, a coin $c_{2}$ is flipped with probability $\tau / \tau^{\prime}$ head. If $c_{2}$ tails, the rejecting algorithm moves on to the next element in $R S$. Otherwise ( $c_{2}$ heads), it decreases the counter $c$ by 1 , and then repeatedly throws a coin $c_{3}$ with probability $1 / \tau^{\prime}$ head, until $c_{3}$ turns on tail. On each head occurrence of $c_{3}$, the counter $c$ is further reduced by 1 , until the element $\{t, c\}$ is eliminated when $c$ equals 0 . At the end of a rejecting pass (after processing all elements), the value of $\tau$ is updated to $\tau^{\prime}$. If the overflow of $R S$ persists (i.e., no counter became 0 in the previous pass), another pass is executed. This process is repeated until the overflow is remedied.

Deleting a tuple $t$ is much easier. The deletion is ignored if $t$ is not in $R S$. Otherwise, the counter $c$ in the corresponding pair $\{t, c\}$ is decreased, and the pair is removed from $R S$ if $c$ reaches 0 . Note that, unlike delete-at-will, the removal of $\{t, c\}$ does not reduce the size of $R S$, that is, a future sample may still be stored at the original position of the removed entry. It is worth mentioning that the set of tuples in $R S$ is not a random sample set of the current relation $T$. However, it can be converted into one by scanning the entire $R S$ (see [13] for details).

A disadvantage of $C S$ (that restricts the applicability of this method in practice) is that the entire $R S$ must be scanned (sometimes repeatedly) for expunging existing sample(s) in case of overflows. When the size of $R S$ is large, scanning $R S$ may delay processing a large number of subsequently arriving 
records, some of which may need to be discarded from the system after the input buffer becomes full. In this case, it is simply impossible to obtain a truly random sample set. Furthermore, the selection of $\tau^{\prime}$ in the rejecting pass is ad-hoc. Gibbons et al. [13] suggest that $\tau^{\prime}$ should be $10 \%$ higher than the current $\tau$, without, however, providing justification on this choice. In Section 4 we present CS*, an enhanced version of CS that avoids these problems.

Motivated by the shortcomings of reservoir and counting sample, Babcock et al. [5] propose alternative algorithms $^{2}$ for producing random samples in the specific context of "sliding window streams", where tuples are deleted according to the order of their arrival. Since these algorithms are inapplicable to arbitrary sequences of insertions/deletions, we do not discuss them further.

Finally, there exist numerous papers $([4,7,18,20]$ to mention just some recent ones) on applications of sampling to various estimation tasks (e.g., selectivity estimation, clustering, etc.). The solutions in those papers do not compute random samples, and cannot be used to solve Q1 and Q2 queries formulated in Section 1. Recently, some sketch-based algorithms $[9,10,11]$ have been developed to obtain a "probabilistic" random sample set, i.e., the samples may be random with a high probability, but there is no guarantee. We aim at deriving truly random samples in all cases.

\subsection{Sampling the Join Results}

Let $T_{1}$ and $T_{2}$ be two relations, and $\theta$ be a join predicate. Nä̈ve stream join (NSJ) is a straightforward method that maintains random sample sets $R S\left(T_{1}\right)$ and $R S\left(T_{2}\right)$ on $T_{1}$ and $T_{2}$, respectively (e.g., by using the techniques of the previous section). Then, given a Q2 query $q$, it finds the number $n_{q}$ of tuple pairs $\left(t_{1}, t_{2}\right) \in R S\left(T_{1}\right) \times R S\left(T_{2}\right)$ that satisfy $q$, and estimates the query result as $\left(n_{q} \cdot\left|T_{1}\right| \cdot\left|T_{2}\right|\right) /\left(\left|R S\left(T_{1}\right)\right| \cdot\left|R S\left(T_{2}\right)\right|\right)$. This estimation, however, is usually not accurate, because the join between the sample sets of $T_{1}$ and $T_{2}$ typically leads to a very small subset of the actual join result. This phenomenon is caused by the fact [8]

\footnotetext{
2 It is worth mentioning that the sliding-window algorithms in [5] have lower update cost than the proposed approaches. Specifically, those algorithms require $O(1)$ cost for each incoming tuple, while our approaches incur $\mathrm{O}(\log M)$ time, where $M$ is the memory size. This comparison, however, is not fair because the algorithms in [5] utilize the special properties of a sliding-window stream to reduce the update cost, and is not applicable in our problem settings.
} 
that the projection of the join result onto the columns of $T_{1}\left(T_{2}\right)$ is not a random sample set of $T_{1}\left(T_{2}\right)$.

To illustrate this, we use an example similar to that in [8]. Consider that $T_{1}$ and $T_{2}$ have a single attribute $A$ with the following tuples: $T_{1}=\{1,1, \ldots, 1,2\}$, and $T_{2}=\{2,2, \ldots, 2,1\}$. Most probably, a random sample $R S\left(T_{1}\right)$ (or $R S\left(T_{2}\right)$ ) on $T_{1}$ (or $T_{2}$ ) will include only records with $A=1$ (or 2 ). Therefore, the size of $T_{1} \bowtie_{\theta} T_{2}$ (where $\theta$ is $T_{1} . A=T_{2} . A$ ) is estimated as 0 , even though the join actually produces a large number of records.

To solve this problem, Chaudhuri et al. [8] suggest sampling the join result in a two-step manner. The first step joins each tuple $t \in T_{1}$ with the data in $T_{2}$, and records $t . w$ (the weight of $t$ ) as the number of records in $T_{2}$ that qualify $\theta$ with $t$. At the end of this step, the algorithm computes the sum $W$ of the weights of all $T_{1}$-tuples. Then, in the second step, each record $t \in T_{1}$ is re-examined. This time, a coin is thrown with head probability proportional to $t . w$. If the coin tails, $t$ is ignored and the execution proceeds with the next tuple in $T_{1}$. Otherwise (the coin heads), a subset of the records in $t \ltimes_{\theta} T_{2}$ (all the join results produced by $t$ ) is randomly extracted and included into $R S_{\theta}$ (the sample set over the join results). The expected size of this subset is also proportional to t.w (see [8] for details).

The above method is static because subsequent insertions and deletions on $T_{1}$ or $T_{2}$ necessarily affect the weights of individual tuples, and hence, change the probabilities that they should appear in $R S_{\theta}$. Srivastava and Widom [24] develop a similar sampling strategy that can handle updates. Their work, however, is restricted to sliding windows and assumes a priori knowledge about data distributions (records follow either the "age-based" or "frequency-based" model). In Section 5.2, we develop alternative methods without such constraints. Acharya et al. [2] propose the "join synopsis" for obtaining random samples in the special case of foreign-key joins, but their methods are inapplicable to arbitrary join conditions. Other relevant work $[3,9,19]$ focuses on estimating the join sizes without computing samples. 


\section{DYNAMIC RESERVOIR SAMPLING}

In this section, we present the $\mathrm{R}^{*}$ algorithm, an extension of reservoir sampling that supports an arbitrary sequence of insertions and deletions. Section 3.1 discusses the algorithmic details of $\mathrm{R}^{*}$ and Section 3.2 analyzes its characteristics.

\subsection{Algorithm}

$\mathrm{R}^{*}$ maintains an array $R S$ with size $M$, whose value is determined by the amount of available memory. At any time, only a subset of valid records in $R S$ belongs to the current sample set. Each element $R S[i](1 \leq i$ $\leq M)$ is associated with a tag $R S[i]$.valid that equals TRUE if $R S[i]$ is valid, and FALSE, otherwise. Initially, every $R S[i]$.valid equals FALSE, indicating an empty sample set. Insertions are handled in a way similar to the conventional reservoir method. Specifically, for each I-command (let $t$ be the record being inserted), we generate a random integer $x$ in the range $\left[1, n_{I}\right]$, where $n_{I}$ is the total number of insertions processed so far. If $x \leq M$, we place $t$ at the $x$-th position $R S[x]$ of $R S$, and set $R S[x]$.valid to TRUE. Otherwise $(x>M)$, no further action is taken and $t$ is ignored.

To handle a D-command $\{\mathrm{D}, i d\}$, on the other hand, we first check if the tuple with the requested $i d$ belongs to the sample set, namely, whether there exists a number $x(1 \leq x \leq M)$ such that the id of $R S[x]$ equals $i d$, and $R S[x]$.valid $=$ TRUE. If $x$ is found, deletion is completed by simply modifying $R S[x]$.valid to FALSE, without affecting the other elements in $R S$. Figure 1 presents the pseudo-code of $\mathrm{R}^{*}$.

We emphasize several differences between $\mathrm{R}^{*}$ and the reservoir algorithm coupled with delete-at-will (reviewed in Section 2.1). First, although both algorithms generate a random number for each incoming I-command, the upper bound of the random number generated by $\mathrm{R}^{*}$ equals $n_{I}$ (Line 2 of $R^{*}$-insert in Figure 1), as opposed to $|T|$ in reservoir.

Second, whenever a sample is deleted, reservoir wastes an element in array $R S$, that is, the element can no longer be used to hold a sample. Accordingly, the maximum possible sample-set size also decreases, thus wasting an increasingly large part of the memory. $\mathrm{R}^{*}$, on the other hand, does not reduce 
the size of $R S$, i.e., in the future the sample set size can still be as large as permitted by the available memory.

Third, after deleting a sample, the positions of the remaining samples in $R S$ are not important for reservoir (e.g., the algorithm is still correct, even if two samples switch their positions). For $\mathrm{R}^{*}$, however, the remaining samples must be fixed to their original positions. The reason for this will be clear in the correctness proof of $\mathrm{R}^{*}$ in the next section.

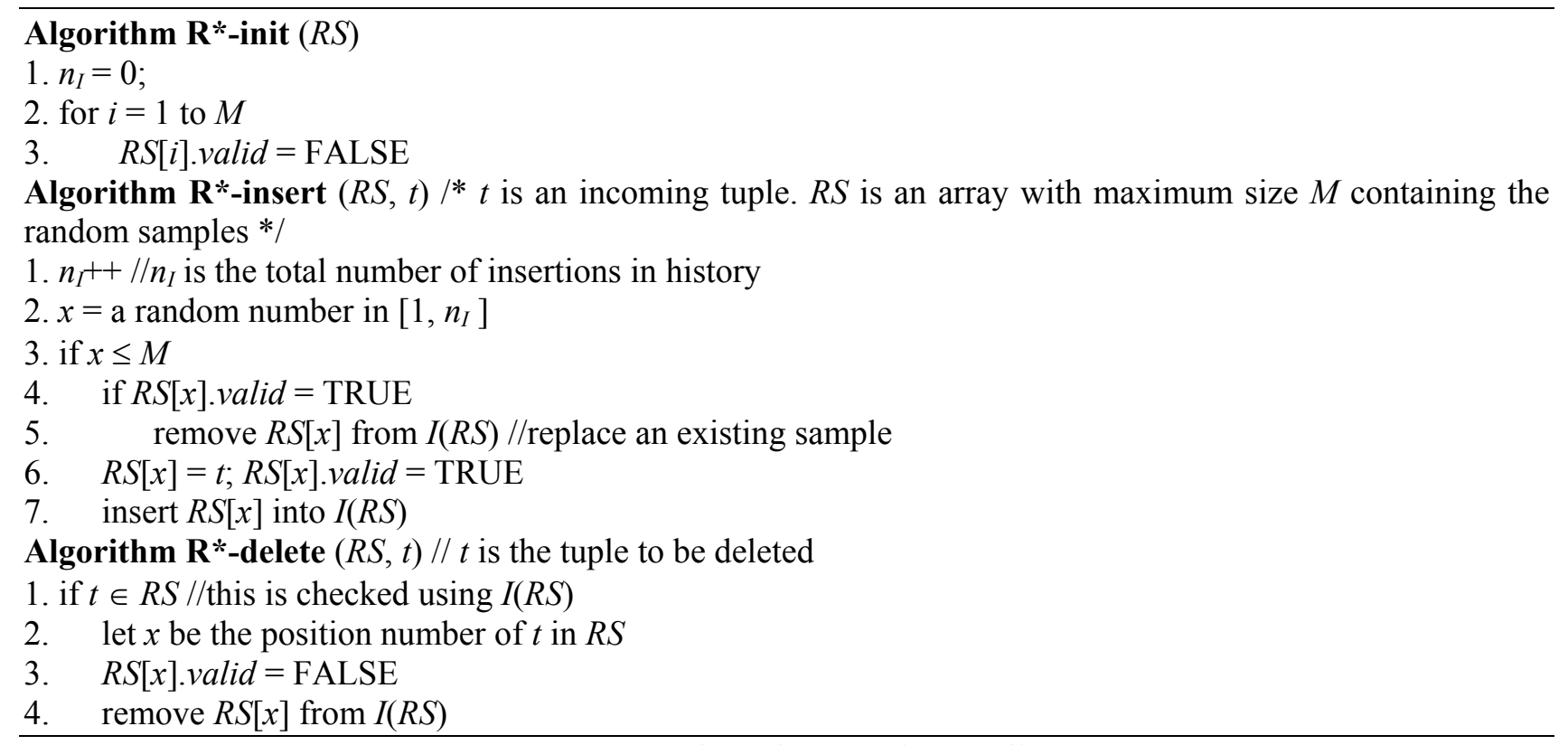

Figure 1: Adapted reservoir sampling

In order to efficiently retrieve records with particular ids (during deletion), we create an appropriate index $I(R S)$ (e.g., a main-memory B-tree [15]) on the sample ids. $I(R S)$ is updated whenever the content of $R S$ changes. Note that, since $I(R S)$ contains only the ids of the tuples, its size is $1 / d$ of the space occupied by the relation, where $d$ is the number of attributes of a tuple.

Given a Q1 query $q$, we count the number $n_{q}$ of valid records in $R S$ that satisfy $q$, and report $n_{q} \cdot T \mid / s$ as the approximate answer, where $|T|$ is the current cardinality of $T$, and $s$ is the number of valid samples. Obviously, both $|T|$ and $s$ can be maintained with trivial overhead - they are simply increased (decreased) whenever a tuple is inserted into (deleted from) $T$ and $R S$, respectively. 


\subsection{Analysis}

We first show that $\mathrm{R} *$ indeed produces a random sample set. Let $U_{\text {seq }}$ be the sequence of all the update requests sorted according to their arrival order, and $I_{i}$ be the $i$-th I-command; similarly, $D_{j}$ is the $j$-th Dcommand. Lemma 1 provides a crucial observation:

Lemma 1. Let $D_{j}$ and $I_{i}$ be a pair of consecutive commands in sequence $U_{\text {seq }}$ such that $D_{j}$ arrives before $I_{i}$ (i.e., $\left.U_{\text {seq }}=\left\{\ldots D_{j} I_{i \ldots}\right\}\right)$. Denote $U_{\text {seq }}^{\prime}$ as the sequence obtained from $U_{\text {seq }}$ by simply swapping the order of $I_{i}$ and $D_{j}$ (i.e., $\left.U_{\text {seq }}^{\prime}=\left\{\ldots I_{i} D_{j} \ldots\right\}\right)$. Then, executing $\mathrm{R}^{*}$ on $U_{\text {seq }}$ and $U_{\text {seq }}^{\prime}$ leads to the same $R S$.

Proof. Since $D_{j}$ arrives before $I_{i}$ in the original sequence $U_{\text {seq }}$, the two updates refer to different records. Let $R S_{b f r}\left(R S_{b f r}^{\prime}\right)$ be the content of $R S$ when all the commands before $D_{j}\left(I_{i}\right)$ in sequence $U_{\text {seq }}\left(U_{s e q}^{\prime}\right)$ have been processed. Obviously, $R S_{b f r}=R S_{b f r}^{\prime}$ since they are the content of $R S$ after processing the same sequence of updates. Similarly, let $R S_{a f t}\left(R S_{a f t}^{\prime}\right)$ be the content of $R S$ after processing $I_{i}\left(D_{j}\right)$ in $U_{s e q}\left(U_{s e q}^{\prime}\right)$. We will show that $R S_{a f t}$ is also identical to $R S_{a f t}^{\prime}$, which establishes the correctness of the lemma, because the remaining parts of $U_{s e q}$ and $U_{s e q}^{\prime}$ are exactly the same.

To prove $R S_{a f t}=R S_{a f t}^{\prime}$, notice that handling a D-command does not generate any random number. Therefore, the value $x$ produced at Line 2 of $R^{*}$-insert (Figure 1) for $I_{i}$ is the same in both $U_{\text {seq }}$ and $U_{\text {seq }}^{\prime}$ (this is why, after deleting a sample, the remaining samples must be kept to their original positions in the array). This indicates that the tuple inserted by $I_{i}$ will appear in both $R S_{a f t}$ and $R S_{a f t}^{\prime}$ simultaneously, or will not appear in any of them. Furthermore, if the tuple requested by $D_{j}$ exists in $R S_{b f r}\left(R S_{b f r}^{\prime}\right)$, it will disappear in $R S_{a f t}\left(R S_{a f t}^{\prime}\right)$, which establishes the correctness of the lemma.

Based on the above lemma, we prove the randomness of the sample set obtained by $\mathrm{R}^{*}$ :

Theorem 1. Let $T$ be the relation being sampled by $\mathrm{R}^{*}$. Then, the valid records in $R S$ always constitute a random sample set for the current content of $T$.

Proof. Assume that the original update sequence $U_{\text {seq }}$ has $n_{I}$ insertions and $n_{D}$ deletions. Let us continuously swap pairs of consecutive D- and I-commands, if the D-command is before the I- 
command. Eventually, we obtain a sequence $U_{\text {seq }}^{\prime}=\left\{I_{1} \ldots I_{n_{I}} D_{1} \ldots D_{n_{D}}\right\}$, where every I-command is positioned before than all the D-commands. Let $R S_{I}$ be the content of $R S$ after performing all the Icommands using the $\mathrm{R}^{*}$ algorithm, which in this case is the same as the conventional reservoir. Hence, $R S_{I}$ is a random sample set over all tuples that have been inserted. The subsequent execution of $\mathrm{R}^{*}$ on the remaining $\mathrm{D}$-commands is reduced to the delete-at-will approach reviewed in Section 2.1, and therefore, the final $R S$ is still a random sample set of $U_{\text {seq. }}^{\prime}$ By Lemma $1, R S$ thus computed is identical to that obtained by running $\mathrm{R}^{*}$ on the original sequence $U_{\text {seq }}$, completing the proof.

Note that the above theorem is correct even if the same object is repeatedly inserted and removed from the database. In this case, special care should be taken to understand semantics of the insertions/deletions in $U_{\text {seq }}^{\prime}$ in the above proof. For example, assume that an object is inserted and removed twice by operation sequence $I_{a}, D_{b}, I_{c}, D_{d}$, for some integers $a<b<c<d$. These 4 updates have order $I_{a}, I_{c}, D_{b}, D_{d}$ in $U_{\text {seq. }}^{\prime}$. Hence, when $D_{b}$ is processed (according to $U_{s e q}^{\prime}$ ), there are two copies of the object in the database (inserted by $I_{a}$ and $I_{c}$, respectively). Then, $D_{b}$ removes the copy created by $I_{a}$, and similarly, $D_{d}$ removes the one created by $I_{c}$.

As a second step, we quantify the number $s$ of valid elements in $R S$ (i.e., the sample size). In particular, our goal is to show that, unlike the delete-at-will approach, the sample size of $\mathrm{R}^{*}$ does not decrease with time, but stabilizes at a certain value depending on the total numbers of insertions and deletions already seen.

Lemma 2. Let $s$ be the number of valid records in $R S$ produced by $\mathrm{R}^{*}$. Then, the probability $P\{s=v\}$ that $s$ equals a particular value $v(1 \leq v \leq M$, where $M$ is the maximum size of $R S)$ is given by:

$$
p\{s=v\}=\left(\begin{array}{c}
n_{D} \\
M-v
\end{array}\right)\left(\begin{array}{c}
n_{I}-n_{D} \\
v
\end{array}\right) /\left(\begin{array}{c}
n_{I} \\
M
\end{array}\right)
$$

where $n_{I}\left(n_{D}\right)$ is the total number of insertions (deletions) processed, assuming $n_{I} \geq M$ (i.e., at least $M$ I- 
commands have been received).

Proof. In the same way as described in the proof for Theorem 1, we convert the original update sequence $U_{\text {seq }}$ into $U_{\text {seq }}^{\prime}=\left\{I_{1} \ldots I_{n_{I}} D_{1} \ldots D_{n_{D}}\right\}$ (the result of $\mathrm{R}^{*}$ on $U_{\text {seq }}$ is equivalent to that on $U_{\text {seq }}^{\prime}$ ). Let $R S_{I}$ be the content of $R S$ after processing all the I-commands. Since $R S_{I}$ is a simple random sample set (with $M$ samples) of the relation $T^{\prime}$ containing the $n_{I}$ inserted records, the number of possible $R S_{I}$ equals $\left(\begin{array}{l}n_{I} \\ M\end{array}\right)$. Furthermore, an $R S_{I}$ leads to a final $R S$ with $v$ valid elements after handling the D-commands in $U_{\text {seq }}$ if and only if two conditions are satisfied. First, $M-v$ (out of $M$ ) tuples of $R S_{I}$ are chosen from the set $T_{D}$ of records deleted by the $n_{D}$ D-commands. Second, the other $v$ tuples of $R S_{I}$ are selected from $T^{\prime}-T_{D}$ (involving $n_{\Gamma}-n_{D}$ records). The number of $R S_{I}$ qualifying the above conditions is $\left(\begin{array}{l}n_{D} \\ M-v\end{array}\right) \cdot\left(\begin{array}{l}n_{I}-n_{D} \\ v\end{array}\right)$.

Therefore, the probability $P\{s=v\}$ can be computed as $\left(\begin{array}{l}n_{D} \\ M-v\end{array}\right) \cdot\left(\begin{array}{l}n_{I}-n_{D} \\ v\end{array}\right) /\left(\begin{array}{l}n_{I} \\ M\end{array}\right)$.

As a corollary, the expected sample size $E(s)$ of $\mathrm{R}^{*}$ equals:

$$
\mathrm{E}(\mathrm{s})=\sum_{v=0}^{M}(v \cdot P\{s=v\})=\sum_{v=1}^{M}(v \cdot P\{s=v\})
$$

where $P\{s=v\}$ is represented in Equation 1. Solving this formula results in the following lemma:

Lemma 3. The expected sample size $E(s)$ equals:

$$
E(s)=M \cdot\left(n_{I}-n_{D}\right) / n_{I}
$$

where $n_{I}$ and $n_{D}$ are as defined in Lemma 2.

Proof. We prove the lemma by induction. First, for $M=1$, Equation 2 becomes $1 \cdot P\{s=1\}$, which, by Lemma 2, is $\left(\begin{array}{c}n_{D} \\ 1-1\end{array}\right) \cdot\left(\begin{array}{l}n_{I}-n_{D} \\ 1\end{array}\right) /\left(\begin{array}{l}n_{I} \\ 1\end{array}\right)=\left(n_{I}-n_{D}\right) / n_{I}$. Hence, the lemma is correct in this case. Next, assuming that the lemma holds for $M$ equal to any integer $k$, we show that it also holds for $M=k+1$.

Let us write $u=v-1$. Hence, Equation 2 can be re-written as (setting $M$ to $k+1$ ):

$$
\sum_{u=0}^{k}(u+1) \cdot\left(\begin{array}{c}
n_{D} \\
k-u
\end{array}\right)\left(\begin{array}{c}
n_{I}-n_{D} \\
u+1
\end{array}\right) /\left(\begin{array}{c}
n_{I} \\
k+1
\end{array}\right)
$$


Since $\left(\begin{array}{l}n_{I}-n_{D} \\ u+1\end{array}\right)=\left(\begin{array}{l}n_{I}-n_{D}+1 \\ u+1\end{array}\right)-\left(\begin{array}{l}n_{I}-n_{D} \\ u\end{array}\right)$ and $\left(\begin{array}{l}n_{I} \\ k+1\end{array}\right)=(k+1) /\left[\left(n_{I}-k\right)\left(\begin{array}{l}n_{I} \\ k\end{array}\right)\right]$, the above equation can be transformed to:

$$
\frac{k+1}{n_{I}-k} \sum_{u=0}^{k}(u+1) \cdot\left(\begin{array}{c}
n_{D} \\
k-u
\end{array}\right) \cdot\left(\begin{array}{c}
n_{I}-n_{D}+1 \\
u+1
\end{array}\right) /\left(\begin{array}{c}
n_{I} \\
k
\end{array}\right)-\frac{k+1}{n_{I}-k} \sum_{u=0}^{k}(u+1) \cdot\left(\begin{array}{c}
n_{D} \\
k-u
\end{array}\right) \cdot\left(\begin{array}{c}
n_{I}-n_{D} \\
u
\end{array}\right) /\left(\begin{array}{c}
n_{I} \\
k
\end{array}\right)
$$

It holds that $\left(\begin{array}{l}n_{I}-n_{D}+1 \\ u+1\end{array}\right)=\frac{n_{I}-n_{D}+1}{u+1}\left(\begin{array}{l}n_{I}-n_{D} \\ u\end{array}\right)$. Furthermore, by the inductive assumption (Lemma 3 holds for $M=k)$, we have $\sum_{u=0}^{k} u \cdot\left(\begin{array}{l}n_{D} \\ k-u\end{array}\right)\left(\begin{array}{l}n_{I}-n_{D} \\ u\end{array}\right) /\left(\begin{array}{l}n_{I} \\ k\end{array}\right)=k \cdot \frac{n_{I}-n_{D}}{n_{I}}$. Combining these facts, we simplify Equation 4 as:

$$
\frac{k+1}{n_{I}-k} \cdot\left(n_{I}-n_{D}\right) \cdot \sum_{u=0}^{k}\left(\begin{array}{c}
n_{D} \\
k-u
\end{array}\right) \cdot\left(\begin{array}{c}
n_{I}-n_{D} \\
u
\end{array}\right) /\left(\begin{array}{c}
n_{I} \\
k
\end{array}\right)-\frac{k+1}{n_{I}-k} \cdot\left(k \cdot \frac{n_{I}-n_{D}}{n_{I}}\right)
$$

Observe that $\sum_{u=0}^{k}\left(\begin{array}{l}n_{D} \\ k-u\end{array}\right)\left(\begin{array}{l}n_{I}-n_{D} \\ u\end{array}\right)=\left(\begin{array}{l}n_{I} \\ k\end{array}\right)$. Hence, the above equation becomes:

$$
\frac{k+1}{n_{I}-k} \cdot\left(n_{I}-n_{D}\right)-\frac{k+1}{n_{I}-k} \cdot\left(k \cdot \frac{n_{I}-n_{D}}{n_{I}}\right)=(k+1) \cdot \frac{n_{I}-n_{D}}{n_{I}} .
$$

Thus, we complete the proof.

The above equation has a clear intuition: The percentage of the valid tuples in $R S$ corresponds to the percentage of the records currently in $T$ among all those ever inserted. Indeed, the probability $P\{s=v\}$ (Equation 1) peaks at $v=M \cdot\left(n_{I}-n_{D}\right) / n_{I}$, and quickly diminishes as $v$ drifts away from this value. This, in turn, indicates a small variance for $s$, thus validating the usefulness of Equation 3. As a corollary of Lemma 3, the actual sample size of $\mathrm{R}^{*}$ is expected to remain constant with time provided that the ratio $n_{I} / n_{D}$ between the numbers of insertions and deletions is fixed.

We are now ready to quantify the per-tuple processing cost of $R^{*}$.

Theorem 2. For each I-command, $\mathrm{R}^{*}$ performs a deletion from $I(R S)$ with probability $E(s) / n_{I}$, and an insertion into $I(R S)$ with probability $M / n_{I}$, where $E(s)$ is the expected sample size given in Equation 2 or 3, $M$ the memory capacity, and $n_{I}$ the total number of insertions. For a D-command, $\mathrm{R}^{*}$ always executes a search in $I(R S)$, and then a deletion from $I(R S)$ with probability $E(s) /\left(n_{I}-n_{D}\right)$. 
Theorem 2 shows that, for each I-/D-command, $\mathrm{R}^{*}$ does nothing with a high probability. When it needs to do something, it performs a single insertion to $I(R S)$ for an I-command, whereas, for a Dcommand, it performs a search and a deletion on $I(R S)$. Since $I(R S)$ is a main-memory B-tree, the worstcase per-tuple cost of $\mathrm{R}^{*}$ is $O(\log |R S|)=O(\log M)$.

\section{Distinct Counting SAMPLe}

In this section, we develop an alternative sampling approach $\mathrm{CS}^{*}$, which is motivated by the counting sample reviewed in Section 2.1, but improves its update performance considerably. The next section first elaborates the sampling procedures of $\mathrm{CS}^{*}$, and Section 4.2 analyzes its behavior.

\subsection{Algorithm}

As with $\mathrm{R}^{*}, \mathrm{CS}^{*}$ maintains an array $R S$ (containing at most $M$ elements), and associates each record $R S[i](1 \leq i \leq M)$ with a tag $R S[i]$.valid to indicate its validity in the current sample set. In addition, it uses a stack, denoted as vacant, to organize the positions of invalid elements. If $R S$ contains $s$ valid tuples, then vacant contains $M-s$ numbers. Let $x$ be the value of vacant $[i](1 \leq i \leq M-s)$; it follows that $R S[x]$.valid must be FALSE. Adopting the idea of the original CS method, CS* deploys a variable $\tau$ to control the probability of sampling an incoming tuple. Before receiving any record, RS[i].valid = FALSE, vacant $[i]$ is set to $i$ (for all $1 \leq i \leq M$ ), and $\tau$ is initialized to 1 .

Consider that an I-command arrives at the system, inserting tuple $t$. CS* throws a coin with probability $1 / \tau$ head (i.e., $1 / \tau$ is the sampling rate), and discards $t$ if the coin tails. Otherwise, it checks whether vacant is empty (equivalently, if all the elements in $R S$ are valid). If not, the position $x=$ vacant $[M-s]$ (i.e., the top of the stack) is obtained, and $t$ is stored at $R S[x]$, which completes the insertion. Now consider the scenario where vacant is empty (i.e., $t$ is not ignored, but $R S$ already contains $M$ valid samples). In this case, a memory overflow occurs, and CS* generates a random number $x$ in the range $[1, M+1]$. If $x=M+1, t$ is expunged, leaving the content of $R S$ intact; otherwise $(x \leq M), t$ is 
placed at the $x$-th element of $R S$, replacing the original sample $R S[x]$. Finally, regardless of the value of $x$, $\tau$ is increased to $\tau \cdot(M+1) / M$ (i.e., the next incoming record is sampled with lower probability).

Processing a D-command (deleting tuple $t$ ) is relatively easy. Specifically, we first check whether $t$ exists in $R S$. If the answer is positive (let $R S[x]=t$ ), $t$ is removed from $R S$ by (i) marking $R S[x]$.valid $=$ FALSE, and (ii) stacking position $x$ into vacant. Similar to $\mathrm{R}^{*}$, to efficiently retrieve $t$ by its id, we maintain an index $I(R S)$ on the ids in $R S$, which is updated with $R S$.

\section{Algorithm CS*-init $(R S)$}

1. $\tau=1 ; s=0$

2. for $i=1$ to $M$

3. $R S[i]$.valid $=$ FALSE; vacant $[i]=i$

Algorithm CS*-insert $(R S, t) / / t$ is an incoming tuple.

1. flip a coin with $1 / \tau$ probability head

2. if the coin heads

3. if $s<M$

4. $x=\operatorname{vacant}[M-s] ; s++$

5. $\quad R S[x]=t ; R S[x]$. valid $=$ TRUE

6. $\quad$ insert $R S[x]$ into $I(R S)$

7. else

8. $x=$ a random number in $[1, M+1]$

9. if $x=M+1$ then ignore $t$

10. else

11. remove $R S[x]$ from $I(R S)$

$12 R S[x]=t$; insert $R S[x]$ into $I(R S)$;

$13 \tau=\tau \cdot(M+1) / M$

Algorithm CS*-delete $(R S, t) / / t$ is the tuple to be deleted

1. if $t \in R S \quad / /$ this is checked using $I(R S)$

2. let $x$ be the position number of $t$ in $R S$

3. $R S[x]$.valid $=$ FALSE; remove $R S[x]$ from $I(R S)$

4. $s--$; vacant $[M-s]=x$

Figure 2: Adapted counting sample algorithm

Figure 2 summarizes the above procedures. Note that, for each I- (D-) command, updating vacant can be done in $O(1)$ time. The query processing algorithm for $\mathrm{CS}^{*}$ is exactly the same as that for $\mathrm{R}^{*}$. Specifically, we count the number $n_{q}$ of valid elements in $R S$ satisfying $q$, and scale up $n_{q}$ by a factor of $|T| / s$. Finally, recall that the stack vacant is not needed in $\mathrm{R}^{*}$ because the position $R S[x]$, where an incoming sample should be stored, is randomly generated even though some records in $R S$ are invalid (i.e., the inclusion of $t$ does not necessarily result in a larger sample size). CS*, on the other hand, always 
uses vacant positions to accommodate new samples (thus increasing the sample size), which requires dedicated structures for recording vacancies ${ }^{3}$.

\subsection{Analysis}

In the sequel, we analyze the performance of $\mathrm{CS}^{*}$, and compare it with the $\mathrm{R}^{*}$ algorithm. Similar to $\mathrm{R}^{*}$, CS* guarantees the randomness of the samples:

Theorem 3. Let $T$ be the stream relation being sampled by $\mathrm{CS}^{*}$. The probability for any tuple in $T$ to be a valid record in $R S$ always equals the current sampling rate $1 / \tau$. As a result, the set of valid elements in $R S$ is a random sample set of $T$.

Proof. We prove the statement by induction. Before the memory overflows for the first time (i.e., $\tau=$ 1), every tuple in $T$ appears in $R S$, in which case the theorem is trivially true. As the inductive step, we assume that the theorem holds after the $k$-th $(k \geq 0)$ overflow, and we show that it is still correct after the next overflow. Let $t$ be the arriving record that causes the $(k+1)$-st overflow, and $t^{\prime}$ be any other tuple in $T$. Then, $t\left(t^{\prime}\right)$ appears in $R S$ after the overflow if (i) the coin heads at line 2 of $C S^{*}$ insert in Figure 2 ( $t^{\prime}$ was already in $R S$ before $t$ arrived), and (ii) it is not discarded (lines 9-12 of $C S^{*}$-insert) during the overflow resolution. The probability for both events to occur equals $(1 / \tau) \cdot M /(M+1)$, which is the adjusted sampling rate after the insertion, thus completing the proof.

At any time, every tuple that has been inserted but not deleted has a probability $1 / \tau$ to be a valid sample in $\mathrm{CS}^{*}$. Therefore:

Lemma 4. The expected sample size of CS* is $\left(n_{I}-n_{D}\right) / \tau$, where $\tau$ is the current sampling rate, and $n_{I}$ $\left(n_{D}\right)$ is the total number of insertions (deletions) that have been processed so far.

A natural question is "which is more accurate: $R^{*}$ or $C S^{*}$ ?". Since both methods return random samples,

\footnotetext{
3 It is worth mentioning that, the stack vacant could be avoided by reorganizing the positions of valid tuples in $R S$ whenever a sample becomes invalid. We do not follow this approach as it leads to considerable maintenance overhead of $I(R S)$.
} 
their accuracy depends solely on the cardinality of their sample sets. Furthermore, notice that the sampling rate of $\mathrm{R}^{*}$ at any time is essentially $M / n_{I}$ which, when multiplied with the current database cardinality $n_{I}-n_{D}$, gives the expected sample set size as in Equation 3. Therefore, in order to compare the sample set sizes of $\mathrm{R}^{*}$ and $\mathrm{CS}^{*}$, it suffices to relate $M / n_{I}$ to the sampling rate $1 / \tau$ of $\mathrm{CS}^{*}$. The comparison result, however, is not definite, i.e., it is possible for either technique to have a larger sample set, depending on the update pattern of the underlying stream. We illustrate this with two concrete examples.

Consider a database whose cardinality $|T|$ remains fixed with time, and assume $|T|>M$. The update sequence consists of $|T|$ initial insertions, after which every subsequent insertion is preceded by a deletion. In this case, the $\mathrm{R}^{*}$ sampling rate $M / n_{I}$ continuously decreases with time due to the growth of the denominator. On the other hand, the sampling rate of CS* remains fixed as soon as the first $|T|$ insertions have been completed, because there is no overflow of array $R S$ after that (recall that CS* decreases its sampling rate by a factor of $M /(M+1)$ only when an overflow occurs). Therefore, eventually, CS* will have a larger sample size than $\mathrm{R}^{*}$.

Assume, on the other hand, that the update sequence contains a large volume $n_{I}$ of insertions, followed by a certain number $n_{D}$ of deletions. Then, the sampling rates of both techniques stabilize after all the insertions are processed. At this moment, CS* has incurred $n_{I}-M$ overflows (in array $R S$ ), leading to a final sampling rate $[M /(M+1)]^{n_{I}-M}$, which can be considerably smaller than the $\mathrm{R}^{*}$ sampling rate $M / n_{I}$ (note that the rate of CS* decays exponentially with $n_{I}$ ). Therefore, after handling all the deletions, $\mathrm{R}^{*}$ will end up with a more sizable sample set.

We summarize the update performance of $\mathrm{CS}^{*}$ with the following theorem.

Theorem 4. For each I-command, CS* performs a deletion and an insertion on $I(R S)$ with probability $1 / \tau$, where $1 / \tau$ is the current sampling rate. For each D-command, a deletion is required with probability $1 / \tau$. 
Similar to $\mathrm{R}^{*}$, by implementing $I(R S)$ as a main-memory B-tree, the per-command processing cost of $\mathrm{CS}^{*}$ is bounded by $O(\log M)$.

\section{SAMPLing METHOdS ON STREAM Joins}

Based on the techniques developed in the previous sections, we proceed to discuss Q2 queries, which return aggregate information about the join of two relations. Section 5.1 presents an algorithm that solves the problem by maintaining random samples on the join results. Then, Section 5.2 proposes an alternative approach with considerably less space consumption and computation overhead.

\subsection{Rigorous Join Sampling}

Recall that all Q2 formulations (on stream relations $T_{a}$ and $T_{b}$ ) possess a common set $\theta_{\text {all }}$ of predicates. We denote $T \bowtie$ as the results of $T_{a} \bowtie_{\theta_{\text {all }}} T_{b}$. Any concrete Q2 instance can be regarded as a Q1 query with its own condition $\theta_{\text {any }}$ on a single relation $T \bowtie$. This observation establishes a natural reduction from problem Q2 to Q1: given a random sample set $R S\left(T_{\bowtie}\right)$ on $T_{\bowtie}$, we can answer any Q2 query in the same way as Q1.

If $T_{a}$ and $T_{b}$ are fully preserved in the system, $R S\left(T_{\rtimes}\right)$ can be maintained using a method (such as $\mathrm{R}^{*}$ or $\mathrm{CS}^{*}$ ) that dynamically computes random samples of a relation. Consider, for example, an arriving Icommand that inserts tuple $t_{a}$ into $T_{a}$. This arrival adds a set of tuples to $T_{\ltimes}$ corresponding to the results of $t_{a} \bowtie_{\theta_{a l l}} T_{b}$ (involving $t_{a}$ and the data in $T_{b}$ ). These tuples are passed to the insertion module of the deployed sampling method for updating $R S(T \bowtie)$. A D-command that removes a tuple $t_{a}$ from $T_{a}$ can be processed in the reverse manner. Specifically, the deletion eliminates from $T_{\bowtie}$ all tuples $t_{a} \bowtie_{\theta_{\text {all }}} T_{b}$, which are fed to the deletion module of the sampling method.

To answer a Q2 query $q$, we count the number $n_{q}$ of samples in $R S(T \bowtie)$ that qualify the condition $\theta_{\text {any }}$

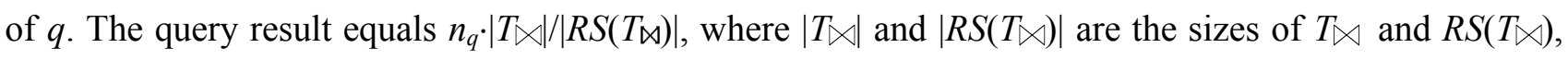
respectively. We call this approach the rigorous join sampling (RJS). Unfortunately, RJS cannot be implemented in stream environments because it requires (i) keeping the entire $T_{a}$ and $T_{b}$ in memory, and 
(ii) examining a complete relation for each update.

Therefore, in the sequel, we focus on approximate solutions that do not have theoretical guarantees, but (i) are scalable to the available amount $M$ of memory, (ii) have low update overhead, and (iii) yet are able to provide accurate answers to Q2 queries.

\subsection{Approximate Join Sampling}

Approximate join sampling (AJS) aims at "simulating" the behavior of RJS. For example, whereas RJS maintains $T_{a}$ and $T_{b}$ completely, AJS preserves only two sets $R S\left(T_{a}\right), R S\left(T_{b}\right)$ of random samples on the two tables (using the $\mathrm{R}^{*}$ or CS* algorithm). Furthermore, as opposed to RJS that outputs a sample set $R S(T \bowtie)$, AJS approximates $R S(T \bowtie)$ with a set $a R S(T \bowtie)$, whose elements are partial pairs of the form $\left\{t_{a}\right.$, $-\}$ or $\left\{-, t_{b}\right\}$, where “-” means NULL, and $t_{a}, t_{b}$ are tuples in $R S\left(T_{a}\right), R S\left(T_{b}\right)$, respectively. Assume, for instance, that the $R S(T \bowtie)$ of RJS currently contains 4 joined pairs $\left\{a_{1}, b_{1}\right\},\left\{a_{1}, b_{2}\right\},\left\{a_{2}, b_{2}\right\},\left\{a_{3}, b_{3}\right\}$. Then, AJS would produce an $a R S(T \bowtie)$ with elements $\left\{a_{1},-\right\},\left\{a_{1},-\right\},\left\{a_{2},-\right\},\left\{-, b_{3}\right\}$. The first pair, for example, corresponds to a join pair (according to $\theta_{\text {all }}$ ) involving $a_{1}$, without indicating the tuple from $T_{b}$ that produces the result. An alternative interpretation of $\left\{a_{1},-\right\}$ is that: any record (e.g., $\left.b_{1}, b_{2}\right)$ in $T_{b}$ qualifying $\theta_{\text {all }}$ with $a_{1}$ can appear in the "-" field with an equal probability. We first explain how to use such incomplete information to answer Q2 queries.

Query algorithm. As discussed earlier, given a Q2 query (with predicate $\theta_{\text {any }}$ ), RJS obtains the number $n_{q}$ of samples in $R S\left(T_{\bowtie}\right)$ that qualify $\theta_{\text {any }}$, and then returns $n_{q} \cdot|T \bowtie|\left|R S\left(T_{\bowtie}\right)\right|$, where $|R S(T \bowtie)|$ and $\mid T_{\bowtie}$ are the cardinalities of $R S\left(T_{\bowtie}\right)$ and $T \bowtie$, respectively. A similar approach is taken by AJS. Specifically, for every partial pair (e.g., $\left\{t_{a},-\right\}$ ) in $\operatorname{aRS}(T \bowtie)$, AJS increases $n_{q}$ by 1 with the probability that the pair satisfies $\theta_{\text {any. }}$. The final result equals $n_{q} \cdot w /|a R S(T \bowtie)|$, where $\mid a R S\left(T_{\bowtie)} \mid\right.$ is the size of $a R S(T \bowtie)$, and $w$ is an estimate for $|T \bowtie|$ (its computation will be clarified later).

Assuming $t_{b}$ to be any tuple in $T_{b}$, the probability that $\left\{t_{a},-\right\}$ satisfies $\theta_{\text {any }}$ equals the conditional probability $P\left\{\theta_{\text {any }} \mid t_{a}, \theta_{\text {all }}\right\}$ that $\left\{t_{a}, t_{b}\right\}$ satisfies $\theta_{\text {any }}$, given that $\left\{t_{a}, t_{b}\right\}$ passes $\theta_{\text {all }}$ (the "given" condition is 
needed for $\left\{t_{a},-\right\}$ to appear in $\left.a R S(T \bowtie)\right)$. We consider that $\theta_{\text {any }}$ and $\theta_{\text {all }}$ are independent, so that $P\left\{\theta_{\text {any }} \mid t_{a}\right.$, $\left.\theta_{\text {all }}\right\}$ is identical to the probability $P\left\{\theta_{\text {any }} \mid t_{a}\right\}$ that $\left\{t_{a}, t_{b}\right\}$ satisfies $\theta_{\text {any. }} . P\left\{\theta_{\text {any }} \mid t_{a}\right\}$ equals the percentage of samples in $R S\left(T_{b}\right)$ qualifying $\theta_{\text {any }}$ with $t_{a}$, and hence, can be easily obtained upon the arrival of $t_{a}$. Figure 3 formally presents the query algorithm based on the above discussion.

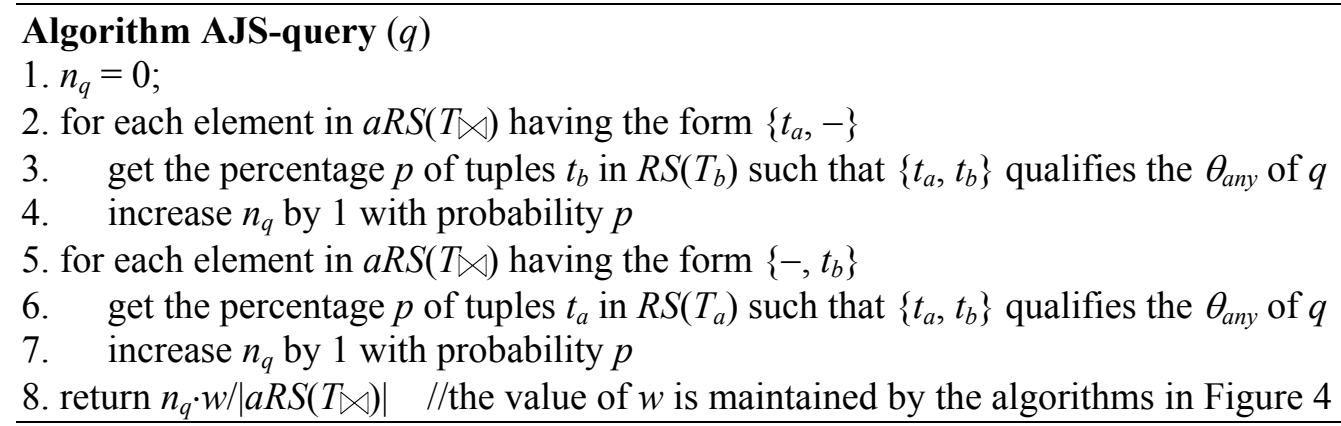

Figure 3: AJS query algorithm

Insertion. We explain how to update $\operatorname{aRS}(T \bowtie)$ assuming that the system has received an I-command inserting tuple $t_{a}$ into $T_{a}$ (the case of inserting into $T_{b}$ is symmetric). Recall that in this case RJS computes $t_{a} \bowtie_{\theta_{\text {all }}} T_{b}$ (the results of joining $t_{a}$ with all tuples in $T_{b}$ ). AJS, on the other hand, simply creates a set of $\left\{t_{a},-\right\}$ pairs to represent the join results. The number of such pairs corresponds to the estimated size of $t_{a}$ $\aleph_{\theta_{\text {all }}} T_{b}$. This estimation reduces to a Q1 query: "How many tuples in $T_{b}$ qualify $\theta_{\text {all }}$ with $t_{a}$ ?". Thus, using the random sample set $R S\left(T_{b}\right)$, the cardinality of $t_{a} \bowtie_{\theta_{a l l}} T_{b}$ can be predicted as $n_{a} \cdot\left|T_{b}\right| /\left|R S\left(T_{b}\right)\right|$, where $n_{a}$ is the number of samples in $R S\left(T_{b}\right)$ satisfying this Q1 query.

Next, RJS will pass the records in $t_{a} \bowtie_{\theta_{\text {all }}} T_{b}$ to the insertion module of a sampling method for updating $R S\left(T_{\rtimes)}\right)$. Accordingly, AJS passes all the $\left\{t_{a},-\right\}$ pairs to the same module for modifying $a R S(T \rtimes)$, i.e., some of $\left\{t_{a},-\right\}$ are sampled into $a R S(T \bowtie)$, while the rest are discarded. Processing the Icommand is completed after the sampling.

Each $\left\{t_{a},-\right\}$ added to $a R S(T \bowtie)$ corresponds to a complete pair $\left\{t_{a}, t_{b}\right\}$ incorporated into $R S(T \bowtie)$ by RJS. Every record $t_{b}$ in $T_{b}$, which qualifies $\theta_{\text {all }}$ with $t_{a}$, has the same probability to appear in the field "-_" of this sampled $\left\{t_{a},-\right\}$. We refer to the probability as "the appearance probability of $\left\{t_{a},-\right\}$ ". 
Specifically, let $n_{\text {sam }}$ be the number of $\left\{t_{a},-\right\}$ taken into $\operatorname{aRS}(T \bowtie)$ (during the processing of the Icommand); the appearance probability equals $n_{\text {sam }} /\left[n_{a} \cdot\left|T_{b}\right| /\left|R S\left(T_{b}\right)\right|\right]$, where the term surrounded by the block parentheses describes the expected number of $T_{b}$ tuples satisfying $\theta_{\text {all }}$ with $t_{a}$ (recall that $n_{a}$ is the number of tuples in $R S\left(T_{b}\right)$ satisfying $\theta_{\text {all }}$ with $t_{a}$, when $t_{a}$ arrives). We associate this probability with every sampled $\left\{t_{a},-\right\}$ (its use will be discussed later).

Deletion. AJS handles deletions also by "following" the corresponding actions of RJS. Given a Dcommand that deletes a record $t_{a}$ from $T_{a}$, RJS will remove from $R S(T \bowtie)$ all the elements involving $t_{a}$. Motivated by this, AJS eliminates the partial pairs in $a R S(T \bowtie)$ that may include $t_{a}$. Such pairs may have the form $\left\{t_{a},-\right\}$ or $\left\{-, t_{b}\right\}$. Since the first case is trivial, we focus on the second one.

Let $\left\{-, t_{b}\right\}$ be a member of $a R S(T \bowtie)$. Resorting to the analogy with RJS, the corresponding "complete" pair in $R S(T \bowtie)$ would include $t_{a}$ only if all the following conditions hold:

1. Tuples $t_{a}$ and $t_{b}$ satisfy $\theta_{a l l}$.

2. Record $t_{a}$ must have arrived before $t_{b}$.

3. When $t_{b}$ was inserted, $t_{a}$ was sampled among all the tuples in $T_{a}$ that qualify $\theta_{\text {all }}$ with $t_{b}$.

Hence, AJS decides to retain $\left\{-, t_{b}\right\}$ if $t_{a}$ and $t_{b}$ violate $\theta_{\text {all }}$. Otherwise, it removes $\left\{-, t_{b}\right\}$ with probability $P_{\text {second }} \cdot P_{\text {third }}$, where $P_{\text {second }}\left(P_{\text {third }}\right)$ is the probability that the second (third) criterion is satisfied. Next, we explain heuristics for obtaining $P_{\text {second }}$ and $P_{\text {third }}$, respectively.

Assume that we know the number $t_{b} . n_{a f t}$ of records in $T_{a}$ that arrived after $t_{b}$, and qualify $\theta_{\text {all }}$ with $t_{b}$. Then, $P_{\text {second }}$ can be approximated as $\left(N_{b}-t_{b} . n_{a f t}\right) / N_{b}$, where $N_{b}$ is the number of tuples currently in $T_{a}$ satisfying $\theta_{\text {all }}$ with $t_{b}$. In particular, $N_{b}$ can be obtained by a Q1 query on the random sample set $R S\left(T_{b}\right)$, with the query predicate derived from $\theta_{a l l}$ and $t_{b}$. Value $t_{b} \cdot n_{a f t}$, on the other hand, can be monitored precisely in a simple way. We only have to set it to 0 when $\left\{-, t_{b}\right\}$ is first included in $a R S(T \bowtie)$. Then, every time a record from stream $T_{a}$ is received, we increase $t_{b} \cdot n_{a f t}$ by 1 if the new record qualifies $\theta_{\text {all }}$ with $t_{b}$ 
It remains to clarify the computation of $P_{\text {third }}$. In fact, it is exactly the "appearance probability of $\left\{-, t_{b}\right\}$ ", computed when tuple $t_{b}$ was inserted. As mentioned earlier, this appearance probability is associated with $\left\{-, t_{b}\right\}$, and thus, does not need to be re-calculated. We summarize the insertion/deletion procedures in Figure 4, which also includes the modification of the estimated size $w$ of $T \bowtie$ (required for query processing), during updates.

\section{Algorithm AJS-init}

1. $\operatorname{aRS}\left(T_{\bowtie}\right)=\varnothing ; R S\left(T_{a}\right)=R S\left(T_{b}\right)=\varnothing$;

2. $S_{a}=S_{b}=\varnothing ; w=0$

Algorithm AJS-insert $\left(t_{a}, X\right)$

${ }^{*} t_{a}$ is a tuple being inserted into $T_{a}$ (the case of inserting a record into $T_{b}$ is symmetric and omitted); $X$ is a random sampling algorithm for single relations $* /$

1. $n_{a}=$ the number of records in $R S\left(T_{b}\right)$ qualifying $\theta_{\text {all }}$ with $t_{a}$

2. $w=w+n_{a} \cdot\left|T_{b}\right| /\left|R S\left(T_{b}\right)\right| \quad / / w$ is the estimated size of $T_{\bowtie}$

3. for $i=1$ to $n_{a} \cdot\left|T_{b}\right| /\left|R S\left(T_{b}\right)\right|$

4. X.insert $\left(\operatorname{aRS}\left(T_{\bowtie}\right),\left\{t_{a},-\right\}\right)$

5. associate each sampled $\left\{t_{a},-\right\}$ with its appearance probability

6. for each record $t_{b} \in S_{b}$ that satisfies $\theta_{\text {all }}$ with $t_{a}$

7. $t_{b} \cdot n_{\text {aft }}++$;

8. X.insert $\left(R S\left(T_{a}\right), t_{a}\right)$

Algorithm AJS-delete $\left(t_{a}, X\right) / * t_{a}$ is a tuple being deleted from $T_{a} * /$

1. $n_{a}=$ the number of records in $R S\left(T_{b}\right)$ qualifying $\theta_{\text {all }}$ with $t_{a}$

2. $w=w-n_{a} \cdot\left|T_{b}\right| /\left|R S\left(T_{b}\right)\right|$

3. for each $\left\{t_{a},-\right\} \in a R S\left(T_{\bowtie}\right)$

4. X.delete $\left(a R S\left(T_{\bowtie}\right),\left\{t_{a},-\right\}\right)$

5. for every record $t_{b} \in R S_{b}$ that satisfies $\theta_{\text {all }}$ with $t_{a}$

6. $\quad n_{b}=$ the number of records in $R S\left(T_{a}\right)$ qualifying $\theta_{\text {all }}$ with $t_{b}$

7. $N_{b}=n_{b} \cdot\left|T_{a}\right| /\left|R S\left(T_{a}\right)\right|$

8. throw a coin $c_{1}$ with probability $\left(N_{b}-t_{b} \cdot n_{a f t}\right) / N_{b}$ head

9. if $c_{1}$ tails then $t_{b} \cdot n_{\text {aft }}-$

10. else

11. throw a coin $c_{2}$ with the appearance probability associated with $\left\{-, t_{b}\right\}$

12. if $c_{2}$ heads

13. X.delete $\left(a R S\left(T_{\bowtie}\right),\left\{-, t_{b}\right\}\right)$

14. X.delete $\left(R S\left(T_{a}\right), t_{a}\right)$

Figure 4: AJS update algorithms

\section{EXPERIMENTS}

We empirically evaluate the effectiveness and efficiency of the proposed methods, using a Pentium IV $\mathrm{CPU}$ at $3 \mathrm{GHz}$. The experimental results are presented in two parts, focusing on Q1 queries in Section 


\section{1, and Q2 in Section 6.2.}

\subsection{Performance of Q1 Processing}

The experiments of this section involve stream relations that have two columns $(i d, A)$. Specifically, the tuples of a stream $T$ are generated according to two parameters dist and $\lambda$. The first parameter dist determines the distribution of $A$-values in the domain $[0,10000]$. Unless specifically stated, we use synthetic data, where dist can be Gau or Zipf (we also include real data towards the end of the subsection). Gau denotes a Gaussian function with mean 5000 and variance 2000, while Zipf corresponds to a Zipf distribution skewed towards 0 with coefficient 0.8 . The second parameter $\lambda$, is an integer that controls the ratio of insertions/deletions in the generated stream. Specifically, each update is an Icommand with probability $\lambda /(\lambda+1)$, or a D-command with probability $1 /(\lambda+1)$, i.e., the chance of receiving an I-command is $\lambda$ times higher than a D-command. An I-command inserts a tuple into $T$ with a unique id, whose $A$-value is determined by dist. A D-command randomly removes a record from the current $T$. For both Gau or Zipf, the entire stream contains 4 million I-commands, while the number of Dcommands is approximately $\lambda$ times lower than that of I-commands.

A Q1 query selects tuples whose $A$-values fall in an interval with length qlen (i.e., its $\theta_{\text {any }}$ is a range condition). The query interval is uniformly distributed in the universe $[0,10000]$. A workload consists of 1000 queries obtained with the same qlen. The relative error of an approximate answer est is defined as $|a c t-e s t| / a c t$, where act is the actual number of records satisfying $\theta_{\text {any }}$. We use two metrics to evaluate accuracy: (i) rele is the average (relative) error of all queries in the workload, and (ii) $\max _{80}$ (called $80 \%$-max error), is the 200-th largest error, that is, the error of the remaining 800 queries $(80 \%$ of the workload) is bounded by $\max _{80}$. While rele indicates the expected accuracy of a technique, $\max \varepsilon_{80}$ reveals its robustness - small $\max _{80}$ means that it is able to capture the results of most queries.

Performance vs. Time. The first set of experiments evaluates the randomness of the samples obtained by $\mathrm{R}^{*}$ and $\mathrm{CS}^{*}$. For this purpose, we assume memory size $M=10000$ (i.e., the sample set can 
accommodate up to $10 \mathrm{k}$ tuples) and streams with $\lambda=4$. After every $400 \mathrm{k}$ I-commands (10\% of the insertions in the stream), we randomly select two sets $S_{R}$ and $S_{C S}$ of tuples from the current $T$, where the cardinality of $S_{R}\left(S_{C S}\right)$ is equivalent to the sample size of $\mathrm{R}^{*}\left(\mathrm{CS}^{*}\right)$ at this time. The rationale is that, if the samples obtained by $\mathrm{R}^{*}\left(\mathrm{CS}^{*}\right)$ are random, they should lead to roughly the same error as $S_{R}\left(S_{C S}\right)$.

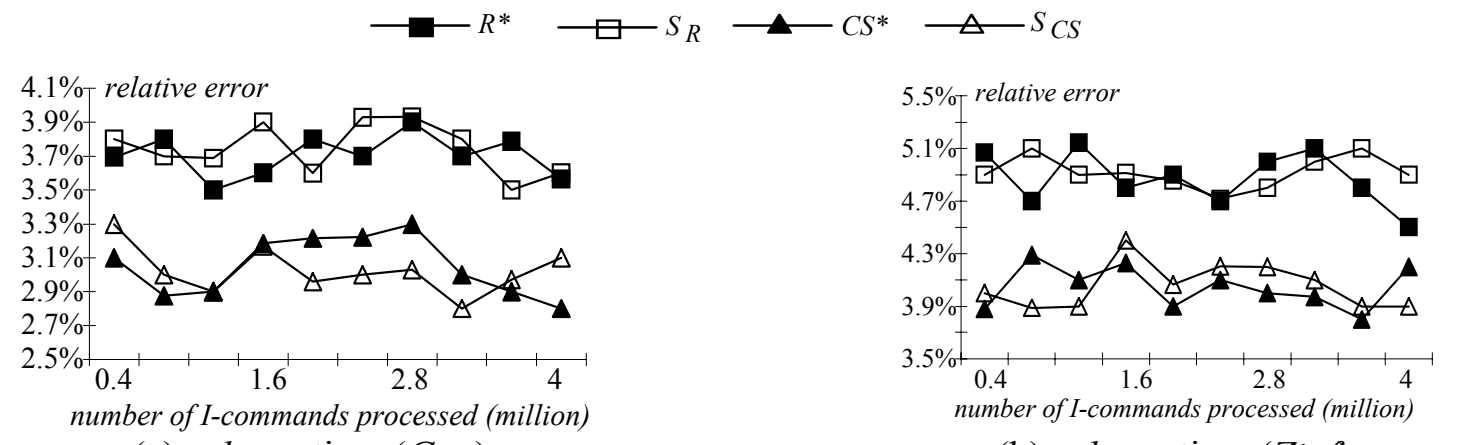

(a) rele vs. time (Gau)

(b) rele vs. time (Zipf)

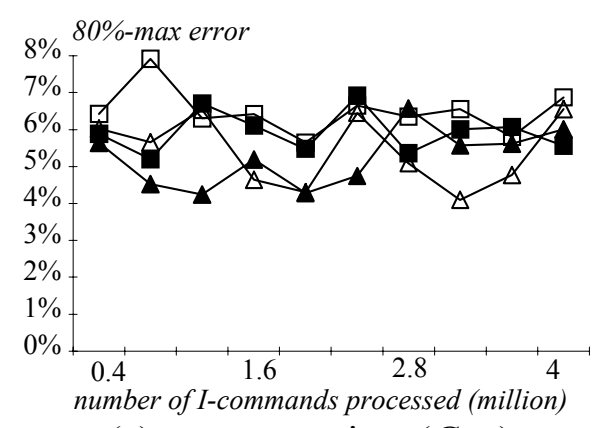

(c) $\max _{80}$ vs. time $(\mathrm{Gau})$

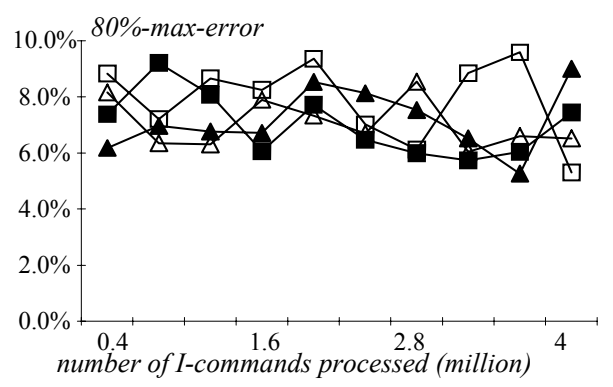

(d) $\max _{80}$ vs. time (Zipf)

Figure 5: Query error verification

Figure 5a (or 5b) shows the rele as a function of the number of I-commands handled for stream Gau (or Zipf) using workloads with qlen $=600$. Figures $5 \mathrm{c}$ and $5 \mathrm{~d}$ illustrate similar results with respect to $\max \varepsilon_{80}$. In all cases, the accuracy of $\mathrm{R}^{*}\left(\mathrm{CS}^{*}\right)$ is statistically similar to that of $S_{R}\left(S_{C S}\right)$. Specifically, the maximum deviation between the rele $\left(\max \varepsilon_{80}\right)$ of $\mathrm{R}^{*}$ and $S_{R}$ is around $0.3 \%(2 \%)$, while the corresponding value for $\mathrm{CS}^{*}$ and $S_{C S}$ is $0.4 \%(1 \%)$. Hence, $\mathrm{R}^{*}$ and $\mathrm{CS}^{*}$ indeed produce random sample sets at the presence of insertions and deletions.

Figure 6 plots the sample size of $\mathrm{R}^{*}$ and $\mathrm{CS}^{*}$ in the experiments of Figures $5 \mathrm{a}$ and $5 \mathrm{c}$ (the results for stream Zipf are omitted due to their similarity). The (sample) sizes remain (almost) constant at $0.75 M=$ $7.5 \mathrm{k}$ tuples for $\mathrm{R}^{*}$ and $M=10 \mathrm{k}$ tuples for $\mathrm{CS}^{*}$. The phenomenon is expected because, as analyzed in 
Sections 3.2 and 4.2 , the sample set size of $\mathrm{R}^{*}$ only occupies a fraction of the available memory (the fraction depends on the ratio between the numbers of insertions and deletions, as shown in Equation 3), whereas CS* is able to utilize all the memory for storing samples. It is known [1] that the relative error of random sampling is inversely proportional to the square root of the sample size. Since the size of $\mathrm{CS}^{*}$ is larger than $\mathrm{R}^{*}$ by $33 \%$, in theory the relative error of $\mathrm{R}^{*}$ is higher by around $15 \%$, which is verified by the results in Figure 5.

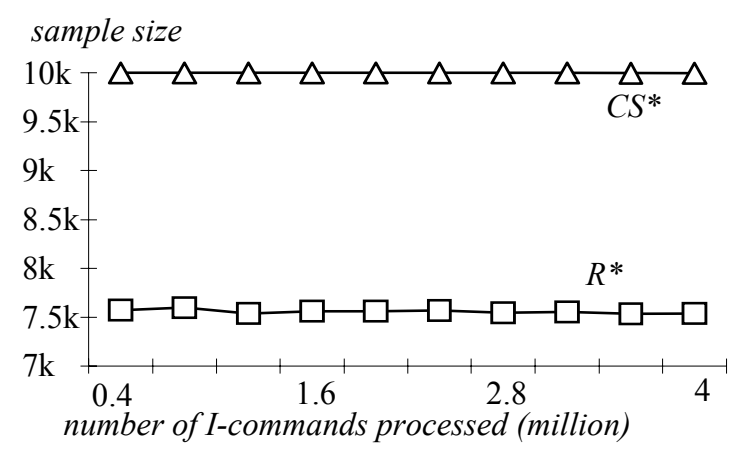

Figure 6: Sample size changes (Gau)

Query Accuracy. As explained in Section 2.1, the (repeated) scanning of the sample set during deletions prevents the application of counting sample to our targeted application domain. Therefore, we use reservoir with delete-at-will as a benchmark since it is the only existing sampling method that incurs small per-record update overhead and can support arbitrary insertions/deletions. The following experiments compare the accuracy of $\mathrm{R}^{*}, \mathrm{CS}^{*}$ and reservoir at the end of streams (recall that the precision of $\mathrm{R}^{*} / \mathrm{CS}^{*}$ remains relatively stable with time).

Figures $7 \mathrm{a}$ and $7 \mathrm{~b}$ illustrate the rele of all algorithms as a function of qlen assuming $M=10 \mathrm{k}$ and $\lambda=$ 4. Clearly, the error of $\mathrm{R} * / \mathrm{CS} *$ is significantly lower than that of reservoir. The better precision for higher qlen is expected as sampling is, in general, more accurate for queries with large output sizes. To study the behavior of alternative techniques under different memory constraints, Figures $7 \mathrm{c}$ and $7 \mathrm{~d}$, measure their precision as a function of $M$, setting qlen and $\lambda$ to 600 and 4 , respectively. Since a larger memory accommodates more samples, the effectiveness of all algorithms increases with $M$. 


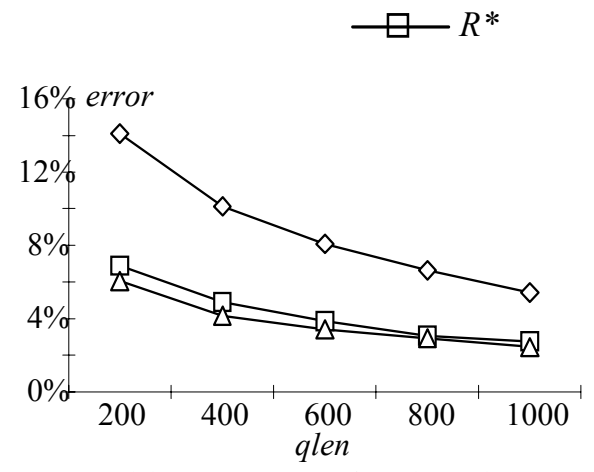

(a) Error vs. qlen (Gau)

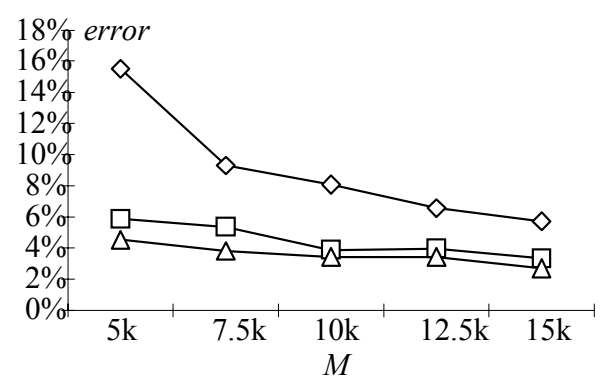

(c) Error vs. $M(\mathrm{Gau})$

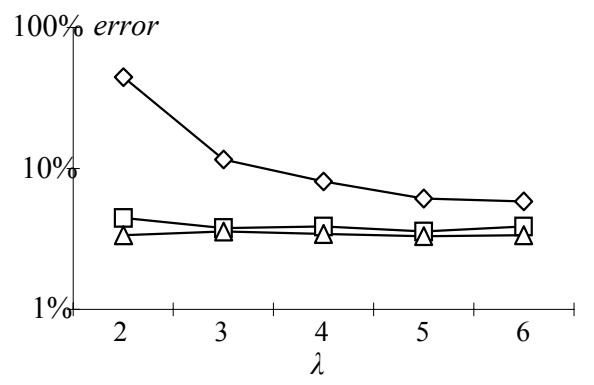

(e) Error vs. $\lambda(\mathrm{Gau})$
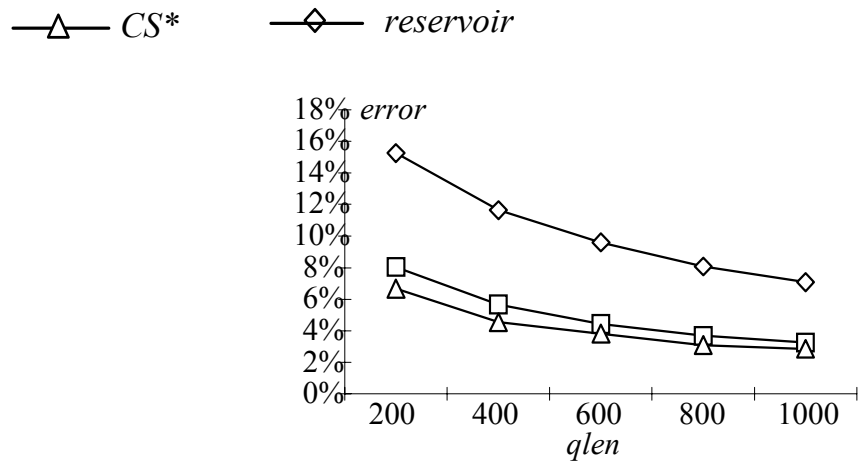

(b) Error vs. qlen (Zipf)

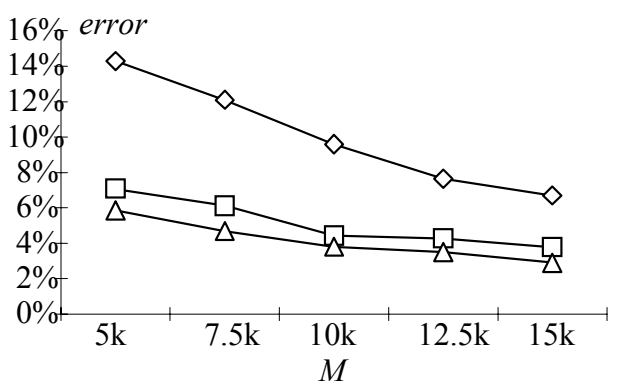

(d) Error vs. $M($ Zipf)

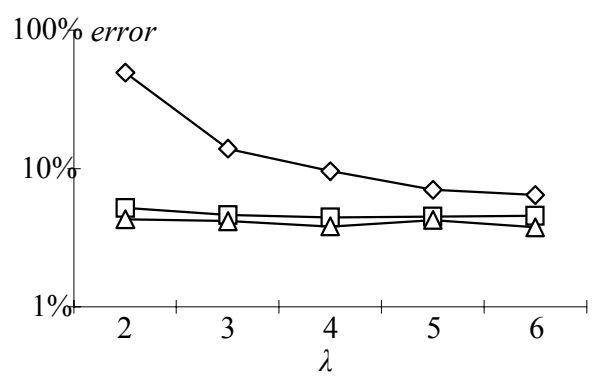

(f) Error vs. $\lambda$ (Zipf)

Figure 7: Query accuracy comparison $($ rele $)$

Figures $7 \mathrm{e}$ and $7 \mathrm{f}$ evaluate $r e l \varepsilon$ for different $\lambda$, after fixing qlen and $M$ to their median values 600 and $10 \mathrm{k}$, respectively. For $\lambda=2, \mathrm{R}^{*}$ and CS* outperform reservoir by more than an order of magnitude (note the logarithmic scale). The performance gap decreases with $\lambda$, because a small number of deletions leads to sample sets with similar cardinalities for all algorithms. Figure 8 repeats the experiments of Figure 7 for $\max _{80}$. In all cases, the results of $\max _{80}$ are similar to rele confirming the robustness of our algorithms. 


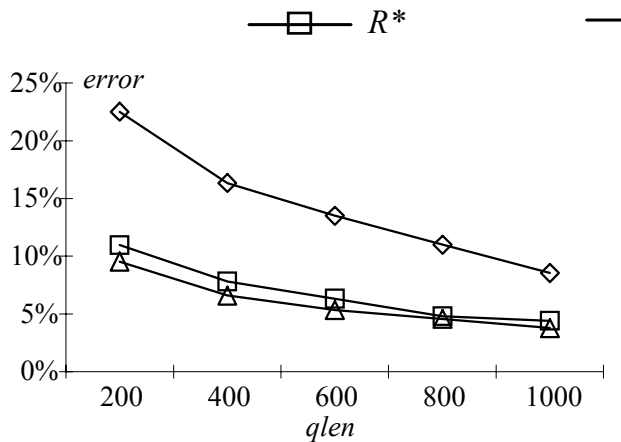

(a) Error vs. qlen (Gau)

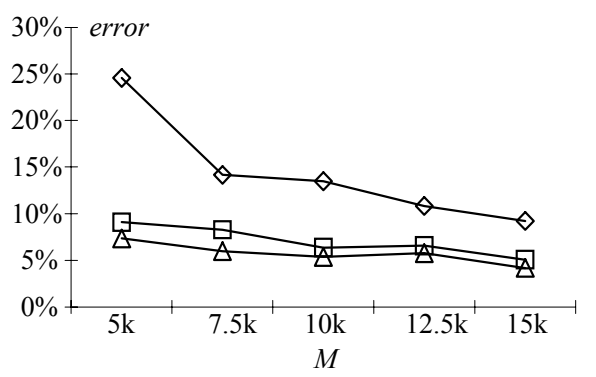

(c) Error vs. $M(\mathrm{Gau})$

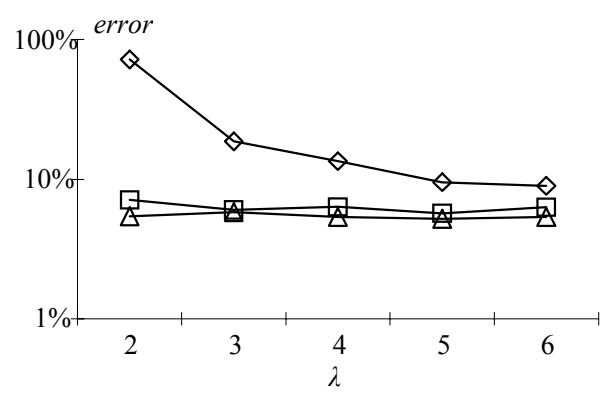

(e) Error vs. $\lambda($ Gau $)$

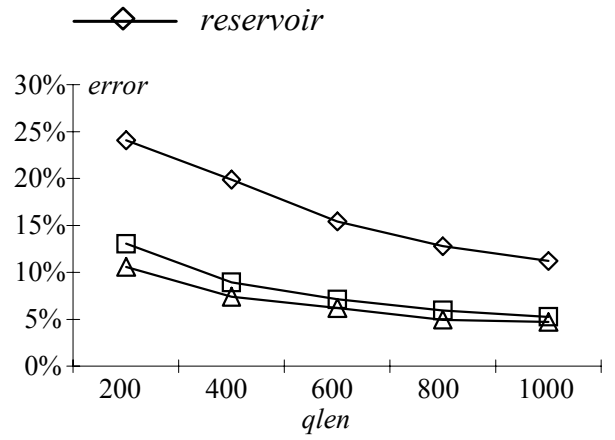

(b) Error vs. qlen (Zipf)

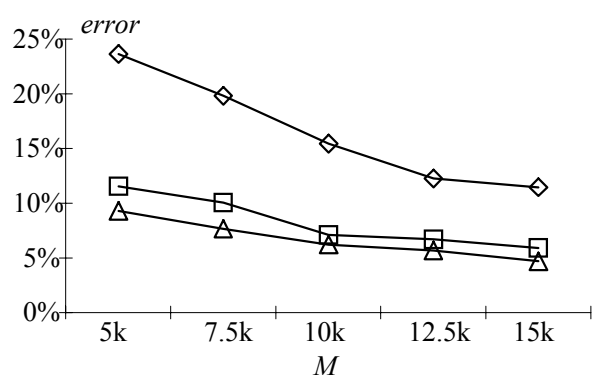

(d) Error vs. $M$ (Zipf)

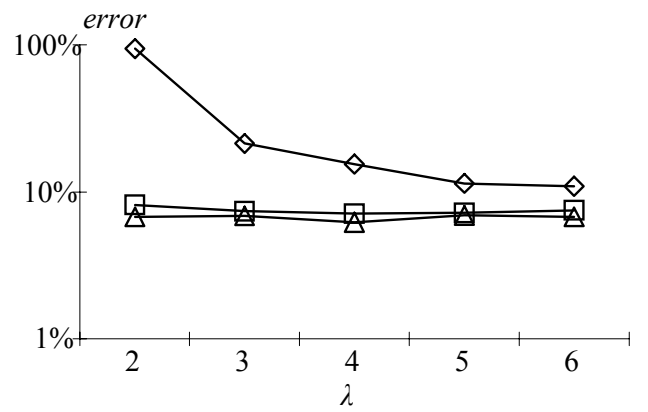

(f) Error vs. $\lambda$ (Zipf)

Figure 8: Query accuracy comparison $\left(\max \varepsilon_{80}\right)$

In order to further verify the generality of our approaches, we also use the real datasets Stock and Tickwise [27]. The former contains the values of 197 North American stocks during the period from 1993 through 1996, and the latter consists of the exchange rates from Swiss francs to US dollars recorded from August 7, 1990 to April 18, 1991. The series Stock (Tickwise) has 512k (279k) values in total. Next, we fix $M$ and $\lambda$ to their median values $10 \mathrm{k}$ and 4 respectively, and measure the accuracy of all solutions at the end of the Stock and Tickwise streams. Figures $9 \mathrm{a}$ and $9 \mathrm{~b}$ compare the rele of different algorithms as a function of qlen for the two distributions respectively, whereas Figures $9 \mathrm{c}$ and $9 \mathrm{~d}$ demonstrate results of $\max \varepsilon_{80}$. Clearly, $\mathrm{R}^{*}$ and $\mathrm{CS}^{*}$ again outperform reservoir considerably in all cases, and their behavior is 
similar to Figures $7 \mathrm{a}, 7 \mathrm{~b}, 8 \mathrm{a}$ and $8 \mathrm{~b}$.

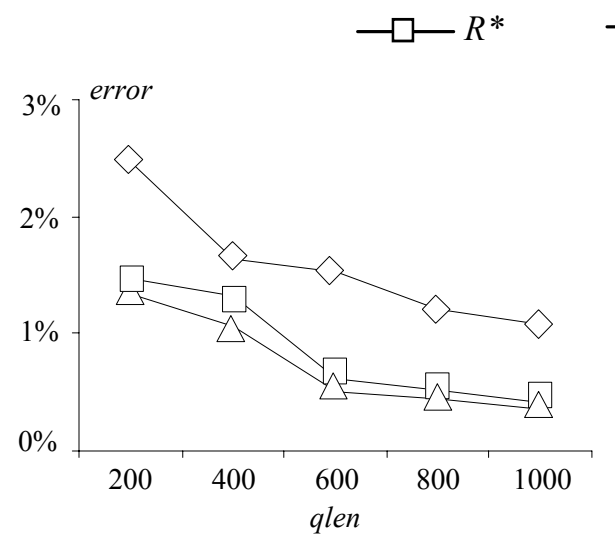

(a) rele vs. qlen (Stock)

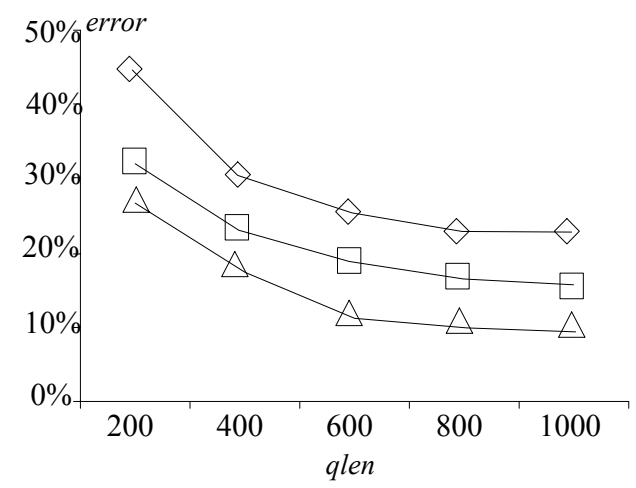

(c) $\max \varepsilon_{80}$ vs. qlen (Stock)

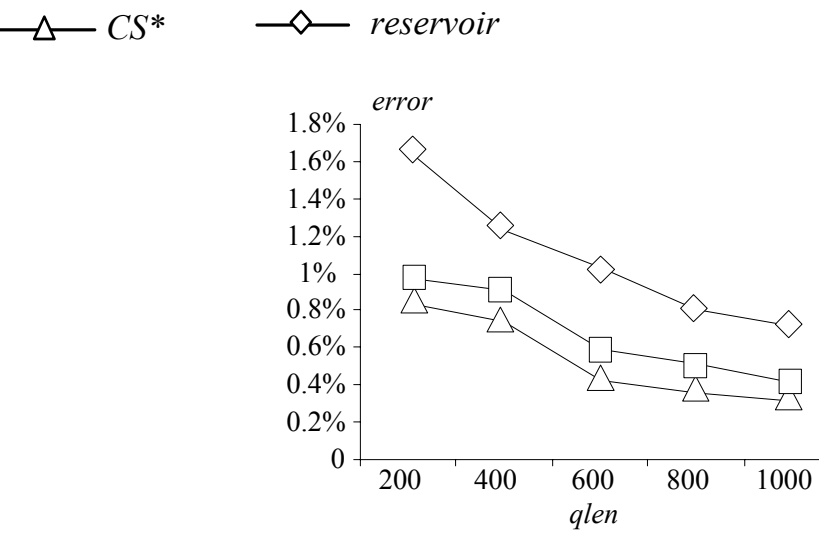

(d) rele vs. qlen (Tickwise)

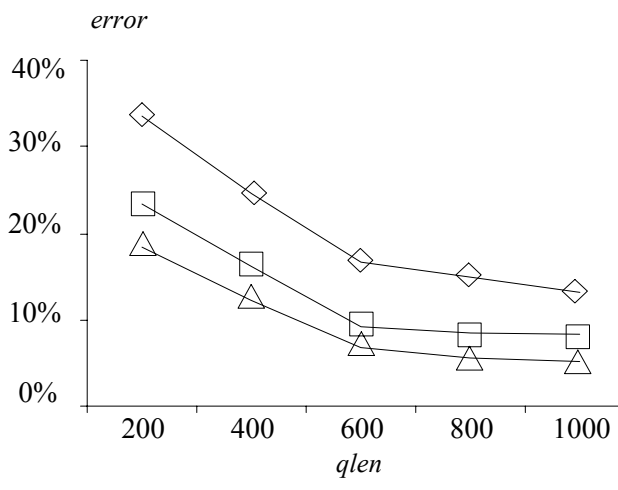

(d) $\max \varepsilon_{80}$ vs. qlen (Tickwise)

Figure 9: Query accuracy comparison (real data)

Update Overhead. Finally, we examine the maintenance overhead of $\mathrm{R}^{*}$ and $\mathrm{CS}^{*}$, which, as explained in Sections 3.2 and 4.2, is dominated by the cost for maintaining $I(R S)$ (i.e., the index on the sample set). In order to provide results independent of index implementation, we measure the total number of times that $I(R S)$ is modified throughout the history (in all cases, the per-tuple cost is so small that it is not even measurable). For instance, $R^{*}-I$-ins and $R^{*}-I$-del denote the number of index insertions and deletions incurred by $\mathrm{R}^{*}$ for processing I-commands. $R^{*}-D$-del corresponds to the number of index deletions for handling D-commands. Figure 10 illustrates these numbers for $\mathrm{R}^{*}$ and $\mathrm{CS}^{*}$ as a function of the memory size for Zipf (the overhead is the same for all distributions). In accordance with Theorems 2 and 4, both methods require higher update costs to produce larger sample sets. $\mathrm{R}^{*}$ incurs about $20 \%$ fewer modifications than $\mathrm{CS}^{*}$ for each type of $I(R S)$ updates due to its smaller sample size. 


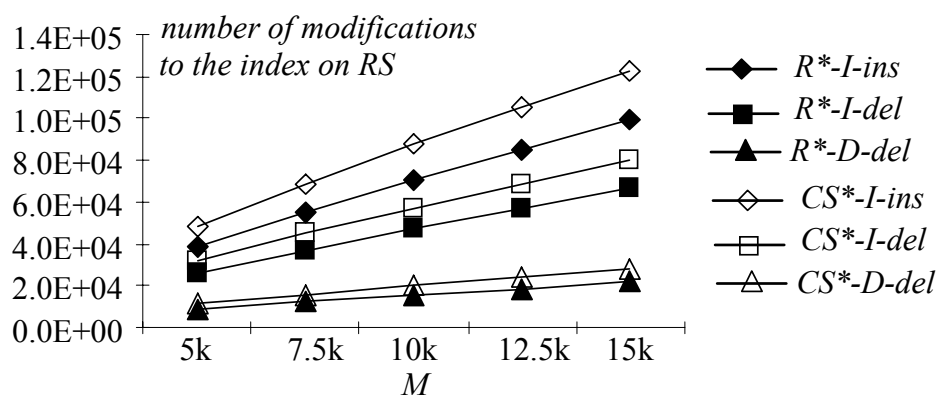

Figure 10: Update cost comparison (Zipf)

\subsection{Performance of Q2 Processing}

Having established the effectiveness of $\mathrm{R} * / \mathrm{CS} *$ for Q1 queries, we proceed to evaluate the efficiency of AJS for Q2 processing. The participating streams $T_{1}$ and $T_{2}$ contain columns $(i d, A, B)$ and $(i d, A, C)$, respectively. The domains of attributes $A, B$, and $C$ are $[0,10000]$. The tuples of each relation are generated in a way similar to the previous section. Specifically, the $A$-values of $T_{1}\left(T_{2}\right)$ follow the Zipf distribution with skew coefficients 0.8 , and the $B-(C$-) values are decided according to a Gaussian function with mean 5000 and variance 2000 . The probability of receiving an I-command is $\lambda=4$ times higher than that of a D-command. Furthermore, streams $T_{1}$ and $T_{2}$ are equally fast, i.e., the next tuple belongs to either relation with equal likelihood.

We consider two scenarios that differ in the way the $A$-values in $T_{1}$ and $T_{2}$ are skewed. Specifically, in the first case skew-skew, the $A$-values in both $T_{1}$ and $T_{2}$ are skewed towards 0 in the domain of [0, 10000]. In the second case anti-skew, data of $T_{1}$ are still skewed towards 0 , while those of $T_{2}$ are towards 10000 - the dense areas of $T_{1}$ and $T_{2}$ are opposite.

Each query involves two conditions. The first one $\theta_{\text {all }}$, common to all (1000) queries in the same workload, has the form $\left|T_{1} \cdot A-T_{2} \cdot A\right| \leq$ len $_{\text {all }}$, where len $_{\text {all }}$ is a workload parameter. The second condition $\theta_{\text {any }}$ includes two range predicates on $T_{1} \cdot B$ and $T_{2} . C$, extracting the tuples of $T_{1}$ and $T_{2}$ falling in these intervals, respectively. Specifically, each range has length len $_{\text {any }}$ (another parameter), and is uniformly distributed in the universe $[0,10000]$. Queries in a workload have different $\theta_{\text {any. }}$ Similar to Section 6.1, 
we quantify the precision of alternative methods by their $r e l \varepsilon$ and $\max _{80}$ for processing a workload. The size of the available memory is fixed to $10 \mathrm{k}$ samples.

Tuning AJS. Recall that AJS consumes a percentage $\eta$ of the memory for keeping two sample sets on $T_{1}$ and $T_{2}$, respectively. The next experiment identifies the value of $\eta$ that achieves the best results. Towards this, we measure the error of AJS in answering a workload with $\operatorname{len}_{\text {all }}=10$ and $\operatorname{len}_{\text {any }}=600$, after all updates of streams $T_{1}$ and $T_{2}$ have been processed. Figure 11a (11b) demonstrates the rel $\varepsilon$ and $\max \varepsilon_{80}$ for the skew-skew (anti-skew) distribution, as a function of $\eta$, including both $\mathrm{R}^{*}$ and $\mathrm{CS}^{*}$ as the underlying sampling algorithms (recall that AJS can be integrated with any sampling technique on individual relations).

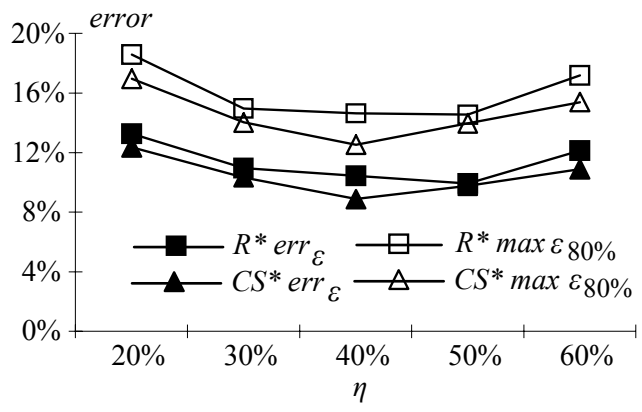

(a) Error vs. $\eta$ (skew-skew)

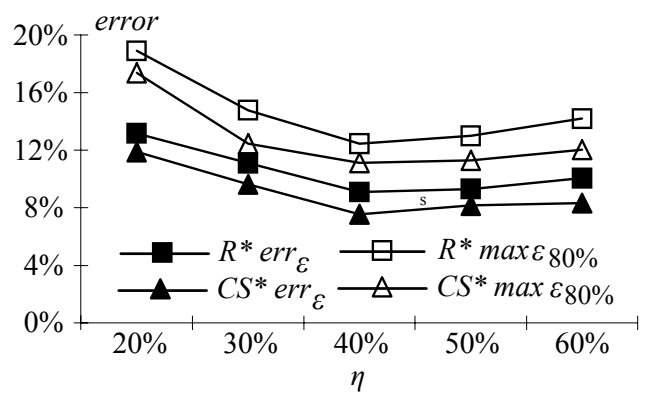

(b) Error vs. $\eta$ (anti-skew)

Figure 11: AJS memory allocation tuning

The accuracy of AJS initially improves as $\eta$ increases and then deteriorates, i.e., the quality of approximation is compromised when too much or little memory is assigned to the random samples on individual relations. A very small $\eta$ prevents correct estimation of the result size of $T_{1} \bowtie_{\theta_{\text {all }}} T_{2}$ (i.e., variable $w$ in Figures 4 and 5), leading to biased results. On the other hand, an excessive $\eta$ leaves limited space for the approximate sample set $a R S(T \bowtie)$ on the join results. In the sequel, we set $\eta$ to $40 \%$, which constitutes a good trade-off. Furthermore, we use $\mathrm{CS}^{*}$ as the representative single-relation algorithm of AJS.

Query Accuracy. Due to the absence of previous methods for sampling join results under tight memory footprint, we compare AJS with the NJS method (see Section 2.2), which maintains random sample sets 
(using $\mathrm{CS}^{*}$ ) on the base relations and produces approximate answers by joining the two sets. Figures 12a and $12 \mathrm{~b}$ compare the error of AJS and NJS as a function of len all, setting $l e n_{a n y}$ to 600 . Clearly, for both skew-skew and anti-skew, AJS outperforms NJS significantly in terms of accuracy and robustness. To study the influence of $l e n_{a n y}$, we set $l e n_{\text {all }}$ to its median value 10 , and repeat the above experiments by varying $l e n_{a n y}$ from 200 to 1000. As shown in Figures 12c and 12d, AJS again outperforms NJS considerably.

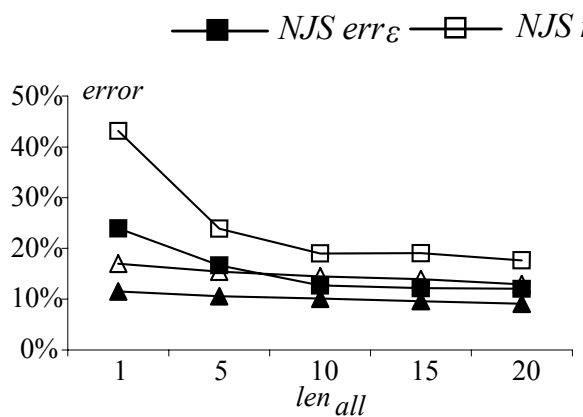

(a) Error vs. qlen all $($ skew-skew)

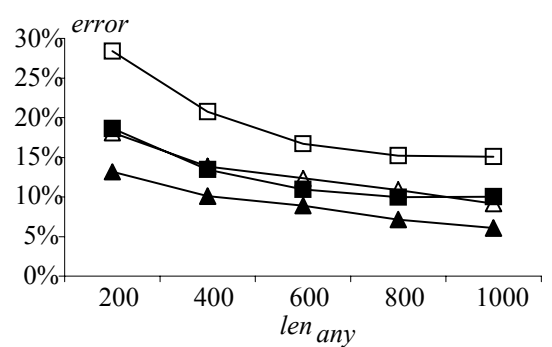

(c) Error vs. qlen any $($ skew-skew)

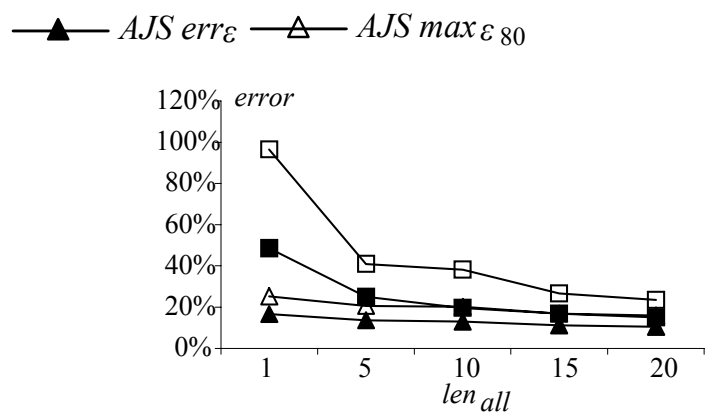

(b) Error vs. qlen all (anti-skew)

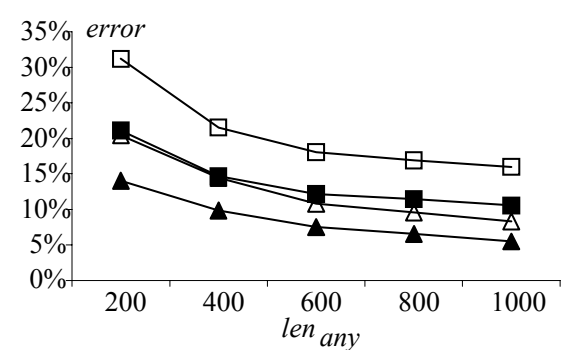

(d) Error vs. qlen any (anti-skew)

Figure 12: Query accuracy comparison

\section{Conclusions}

This paper presents the first random sampling algorithms, $\mathrm{R}^{*}$ and $\mathrm{CS}^{*}$, for efficiently computing aggregate data over streams of arbitrary updates. Going one step further, we propose AJS, a method for sampling join results. We prove, both theoretically and empirically, that our techniques provide accurate answers with small space and computational overhead. While our current focus is on sample sets that fit in the main memory, we plan to investigate situations where part of the sample set is migrated to the disk [21]. Further, it has been observed [6] that the performance of sampling (for approximate aggregate processing) can be improved if query statistics are available in advance. The design of such "workload- 
aware" methods in stream environments constitutes an interesting topic. Finally, we would like to explore the applicability of random sampling for tracking the positions of continuously moving objects [23]).

\section{ACKNOWLEDGEMENTS}

Dimitris Papadias was supported by Grant HKUST 6184/05E from the Research Grant Council of the HKSAR government. We would like to thank the anonymous reviewers for their insightful comments.

\section{REFERENCES}

[1] S. Acharya, P. B. Gibbons, V. Poosala. Congressional samples for approximate answering of group-by queries. SIGMOD, 2000.

[2] S. Acharya, P. B. Gibbons, V. Poosala, and S. Ramaswamy. Join synopses for approximate query answering. SIGMOD, 1999.

[3] N. Alon, P. B. Gibbons, Y. Matias, and M. Szegedy. Tracking join and self-join sizes in limited storage. In PODS, 1999.

[4] B. Babcock, S. Chaudhuri, and G. Das. Dynamic sample selection for approximate query processing. SIGMOD, 2003.

[5] B. Babcock, M. Datar, and R. Motwani. Sampling from a moving window over streaming data. In Proc. of the Annual ACM-SIAM Symposium on Discrete Algorithms, 2002.

[6] S. Chaudhuri, G. Das, and V. Narasayya. A robust, optimization-based approach for approximate answering of aggregate queries. SIGMOD, 2001.

[7] S. Chaudhuri, G. Das, and U. Srivastava. Effective use of block-level sampling in statistics estimation. In SIGMOD, 2004.

[8] S. Chaudhuri, R. Motwani, and V. Narasayya. On random sampling over joins. SIGMOD, 1999.

[9] G. Cormode, S. Muthukrishnan, and I. Rozenbaum. Summarizing and mining inverse distributions on data streams via dynamic inverse sampling. VLDB, 2005.

[10] G. Frahling, P. Indyk, and C. Sohler. Sampling in dynamic data streams and applications. Annual symposium on computational geometry, 2005.

[11] S. Ganguly, M. Garofalakis, and R. Rastogi. Processing set expressions over continuous update streams. SIGMOD, 2003.

[12] S. Ganguly, P. B. Gibbons, Y. Matias, and A. Silberschatz. Bifocal sampling for skew-resistant join size estimation. SIGMOD, 1996.

[13] P. B. Gibbons and Y. Matias. New sampling-based summary statistics for improving approximate query answers. SIGMOD, 1998.

[14] P. B. Gibbons, Y. Matias, and V. Poosala. Fast incremental maintenance of approximate histograms. TODS, 27(3):261-298, 2002. 
[15] G. Graefe and P.-A. Larson. B-tree indexes and cpu caches. ICDE, 2001.

[16] S. Guha, C. Kim, and K. Shim. Xwave: Approximate extended wavelets for streaming data. VLDB, 2004.

[17] S. Guha, K. Shim, and J. Woo. Rehist: Relative error histogram construction algorithms. VLDB, 2004.

[18] P. J. Haas and C. Konig. A bi-level bernoulli scheme for database sampling. SIGMOD, 2004.

[19] P. J. Haas, J. F. Naughton, and A. N. Swami. On the relative cost of sampling for join selectivity estimation. PODS, 1994.

[20] C. Jermaine. Robust estimation with sampling and approximate pre-aggregation. VLDB, 2003.

[21] C. Jermaine, A. Pol, and S. Arumugam. Online maintenance of very large random samples. SIGMOD, 2004.

[22] K.-H. Li. Reservoir-sampling algorithms of time complexity o(n $(1+\log (\mathrm{n} / \mathrm{n})))$. ACM Trans. Math. Softw., 20(4):481-493, 1994.

[23] M. F. Mokbel, X. Xiong, and W. G. Aref. Sina: scalable incremental processing of continuous queries in spatio-temporal databases. SIGMOD, 2004.

[24] U. Srivastava and J. Widom. Memory-limited execution of windowed stream joins. VLDB, 2004.

[25] N. Tatbul, U. Cetintemel, S. B. Zdonik, M. Cherniack, and M. Stonebraker. Load shedding in a data stream manager. VLDB, 2003.

[26] J. S. Vitter. Random sampling with a reservoir. ACM Trans. Math. Softw., 11(1):37-57, 1985.

[27] (Web) Keogh, E. (2006). The UCR Time Series Data Mining Archive (http://www.cs.ucr.edu/ eamonn/TSDMA/index.html). Riverside CA. University of California Computer Science \& Engineering Department. 